\title{
Species Richness and Community Structure of Amphibians and Reptiles in Andanan Watershed Forest Reserve, Caraga Region, Philippines
}

\author{
Chennie L. Solania I,", Jerry T. Cuadrado², Adam Roy V. Galolo' \& Eve F. Gamalinda' \\ ${ }^{1}$ Department of Biology, College of Mathematics and Natural Sciences, Caraga State University, Ampayon, Butuan \\ City, 8600 Caraga, Philippines \\ ${ }^{2}$ Surigao State College of Technology, Surigao City, 8400 Caraga, Philippines \\ *Corresponding author, email: 888cheny@gmail.com
}

\begin{abstract}
The Andanan watershed is a protected natural forest reserve of the Philippines located in the Municipality of Sibagat and City of Bayugan, Caraga Region. This study conducted an extensive opportunistic survey that yielded 49 species of herpetofauna ( 22 frogs, 5 agamids, 4 gekkonid lizards, 7 skinks, one varanid, 9 snakes, and a freshwater turtle), accounting for $67.35 \%$ endemicity in the area. Five habitat types were recorded with a gamma diversity of $H^{\prime}=3.093$. The secondary growth forest was statistically significant at $\mathrm{P}<0.0001$ and gained the highest in species diversity $\left(\mathrm{H}^{\prime}=2.654\right)$. The old-growth forest harbored a distinct species composition with some species not recorded in other habitat types. Six of the endemic amphibians were associated with high canopy cover, while edible amphibians were associated with open and cleared areas. Eleven out of 19 endemic reptiles were often observed in areas with high air temperature, while most of the snakes were observed in old forest stands. The secondary growth forest's structural complexity provides habitat to many herpetofauna, but the uniqueness of the old-growth forest also supports habitat to pristine forest specialists. The results of habitat associations are relevant in promoting local conservation of the herpetofauna and the ecosystem. The area's high conservation value and the apparent threats (forest degradation and resource extraction) necessitates urgent attention and monitoring.
\end{abstract}

KEY WORDS Andanan Watershed Forest Reserve; community structure; habitat association; habitat types; Herpetofaunal composition.

Received 10.02.2021; accepted 28.06.2021; published online 14.09.2021

\section{INTRODUCTION}

In the Philippines, many regions support unique herpetofaunal assemblages (Diesmos \& Brown, 2009; Brown et al., 2013; Sanguila et al., 2016) recognizing the country as one of the most important centers of herpetofaunal diversity and endemism in Southeast Asia (Diesmos et al., 2002). The country hosts 112 species of amphibians with $83.9 \%$ endemics (Diesmos et al., 2015) and 361 species of reptiles (Uetz et al., 2018) with 66\% endemic species (Gojo-Cruz et al., 2019). In one of the three major islands of the country, Mindanao, the efforts on herpetofaunal surveys were concentrated in the southern and western regions only (Delima et al., 2007; Beukema, 2011; Relox et al., 2011; Nuñeza et al., 2016). Surveys in the northeastern part of Mindanao (Caraga Region) includes the work of Calo \& Nuñeza (2015) and Sanguila et al., (2016). Many forested areas and watersheds are still deficient or lacking in baseline information on herpetofaunal diversity. One recently published study in Caraga Region focused only on the anurans of one specific area situated in AWFR (Solania \& Gamalinda, 2018). 
The Andanan Watershed Forest Reserve (AWFR) is one of the largest of the 12 protected areas in the Caraga Region with a total of 15,097 hectares as declared by the Department of Environment and Natural Resources of the Philippines (FMB-DENR, 2013). AWFR offers various ecosystems services such as forest products, water source for irrigation and consumption and as habitats to a unique assemblage of flora and fauna. Numerous caves, waterfalls, and rock formations composed the landscape of the protected area potentially supporting variety of organisms (Mindanao Protected Area Network, 2018). However, settlements and land claims are proliferating in the area and agriculture seems to ascend in upper elevations. It was observed that several forest habitats are converted into farmlands such as falcata and banana plantations which are alarmingly increasing. These anthropogenic activities lead to habitat degradation, destruction, and alteration that may threaten the forest-dependent organisms in the watershed, such as the amphibians and reptiles. Forest-dependent organisms are very vulnerable to changes in the ecosystem because they depend so much on the services provided by the forest such as protection from predators, food, refuge and shelter.

Biodiversity research is a central topic in Watershed Forest Reserves' (WFRs) conservation and management. Such an undertaking is essential for the sustainable utilization of WFRs products and management as a whole. Amphibians and reptiles are bio-indicator species due to their susceptibility to environmental changes (Manolis et al., 2002; Sumanasekara et al., 2015). The community structure analysis and knowledge of habitat preferences of the herptiles are crucial in the management and creation of conservation policies for these indicator species (Riyanto, 2011). The protection and conservation of these disturbance-susceptible herpetofauna is important in maintaining the provisioning, regulating, cultural and supporting economic services these group of organisms offer (Valencia-Aguilar et al., 2013).

AWFR is one of the least studied protected areas in the region which may be attributed to security issues and some areas inaccessible to surveys. This study intends to provide a comprehensive record of amphibians and reptiles of the area. Species-environment relationship analysis was also done to identify the habitat association of Philippine endemic herptiles. Moreover, the results of this study can be used as a basis for possible interventions that would lead to the conservation efforts of herpetofauna. Thus, providing input into the watershed management and decision-making tools of the AWFR.

\section{MATERIAL AND METHODS}

\section{Study Area and Establishment of Sampling Stations}

Andanan Watershed Forest Reserve (AWFR) is one of Caraga Region's major forest reserves (Fig. 1). The watershed is characterized by a mosaic of forest blocks with a large mixture of secondary growth forests and plantation forests. AWFR experiences Type II climate, characterized by wet and very wet seasons. The watershed provides social, aesthetic and economic value in the form of wood and other forest products. It supplies irrigation to farmlands and provide potable water to the local community.

Diurnal and nocturnal surveys were conducted from April 2017 to February 2018 in the four sampling stations of AWFR (Table 1 and Figs. 2-5). These sampling stations were identified based on its accessibility and safety; proximity to Andanan River; and the types of vegetation. The sampling localities include Area 1 - Calaitan (C), Area 2 Berseba (B), Area 3 - Mt. Carmel (MC), and Area 4 - San Juan (SJ). Along the sampling stations, five forest types were observed, namely: Secondary Growth Forest (SGF), Cultivated Area (CA), AgroForest Habitats (AFH), Open-Area Habitats (OAH) and Old-Growth Forest (OGF). DENR Gratuitous Permit was secured before the conduct of the study with GP number R13-2017-0050 (for reptiles) and R13-2017-0051 (for amphibians). Three sampling sites with six standardized $10 \mathrm{~m}$ x 100m (Supsup et al., 2016) strip transect with 50 to 100 meters intervals were randomly established in each station cutting across various habitats. A total of 12 sampling sites with 72 transect lines covering 7.2 hectares were established within the watershed forest reserve.

\section{Herpetofaunal Surveys}

The collection of herpetofauna was done using a combination of techniques: transect walk, intensive visual encounter survey or opportunistic sampling, and mark-release-recapture method. All encountered individuals were captured by hand or with the use of mittens and snake tongs. Opportun- 


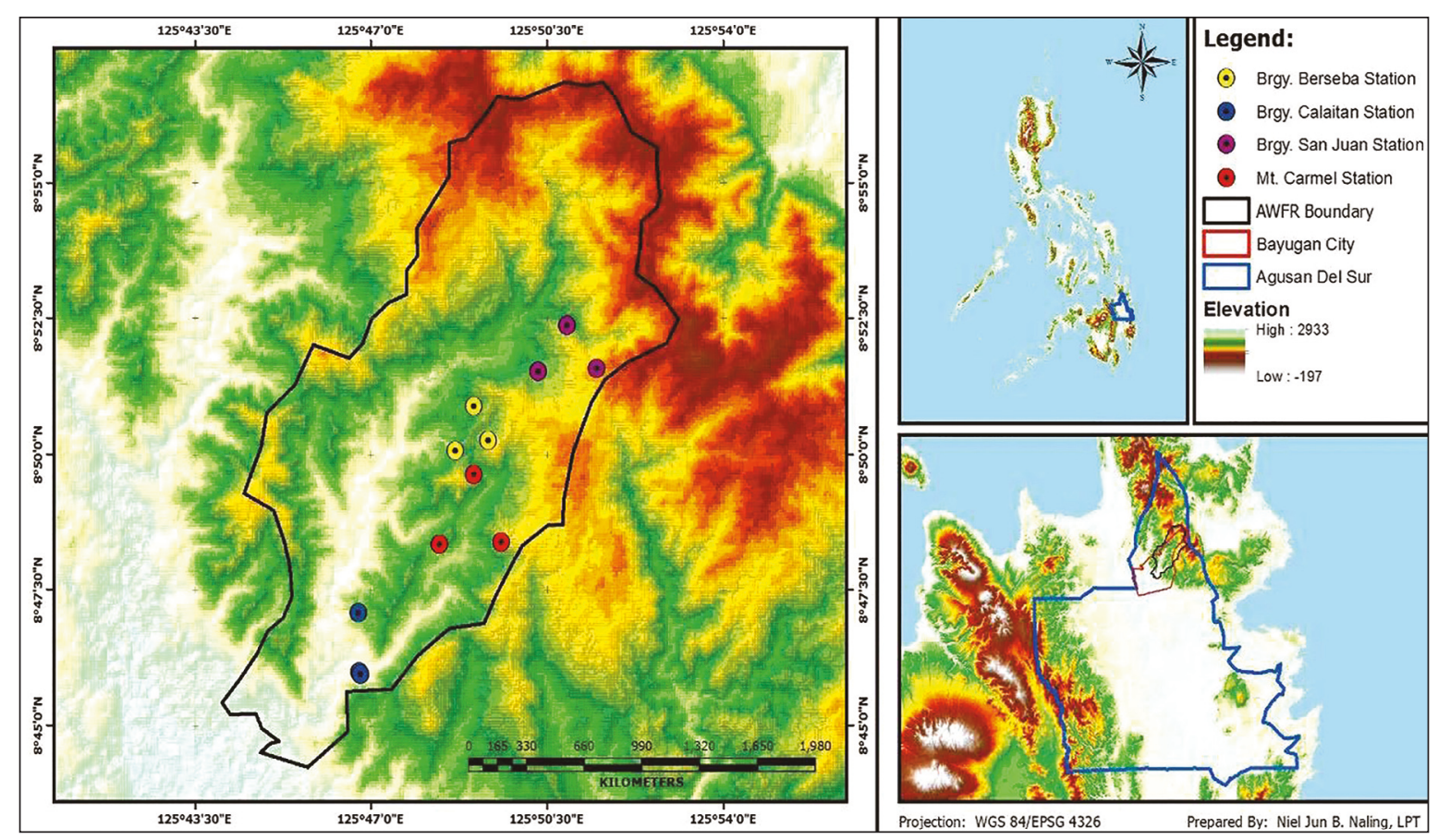

Figure 1. The Philippine map showing the geographic location of Agusan del Sur in Mindanao and the four sampling stations in Andanan Watershed Forest Reserve.

\begin{tabular}{|c|c|c|c|c|c|}
\hline $\begin{array}{c}\text { Sampling } \\
\text { Stations }\end{array}$ & $\begin{array}{c}\text { Sampling } \\
\text { Sites }\end{array}$ & $\begin{array}{l}\text { Latitude } \\
\text { (North) }\end{array}$ & $\begin{array}{c}\text { Longitude } \\
\text { (East) }\end{array}$ & \begin{tabular}{|c|} 
Highest \\
Elevation \\
(masl)
\end{tabular} & $\begin{array}{c}\text { Habitat } \\
\text { Description }\end{array}$ \\
\hline \multirow{3}{*}{$\begin{array}{l}\text { Area 1 } \\
\text { Barangay } \\
\text { Calaitan }\end{array}$} & 1 & $\begin{array}{c}8^{\circ} 45^{\prime} \\
59.6232^{\prime \prime} \\
\end{array}$ & $\begin{array}{c}125^{\circ} 46^{\prime} \\
46.6292^{\prime \prime} \\
\end{array}$ & 118.67 & \multirow{3}{*}{$\begin{array}{c}\text { disturbed lowland, } \\
\text { mixed agricultural } \\
\text { and secondary } \\
\text { forest }\end{array}$} \\
\hline & 2 & $\begin{array}{c}8^{\circ} 45^{\prime} \\
54.3528^{\prime \prime}\end{array}$ & $\begin{array}{c}125^{\circ} 46^{\prime} \\
48.4176^{\prime \prime}\end{array}$ & 142 & \\
\hline & 3 & $8^{\circ} 47^{\prime} 0.5352^{\prime \prime}$ & $\begin{array}{l}125^{\circ} 46^{\prime} \\
42.798^{\prime \prime}\end{array}$ & 215.67 & \\
\hline \multirow{3}{*}{$\begin{array}{l}\text { Area } 2 \\
\text { Barangay } \\
\text { Berseba }\end{array}$} & 4 & $8^{\circ} 50^{\prime} 3.928^{\prime \prime}$ & $\begin{array}{c}125^{\circ} 48^{\prime} \\
40.7628^{\prime \prime}\end{array}$ & 301 & \multirow{3}{*}{$\begin{array}{c}\text { secondary growth } \\
\text { forest and } \\
\text { cultivated areas }\end{array}$} \\
\hline & 5 & $\begin{array}{c}8^{\circ} 50^{\prime} \\
53.0124^{\prime \prime}\end{array}$ & $\begin{array}{l}125^{\circ} 49^{\prime} \\
2.3088^{\prime \prime}\end{array}$ & 460 & \\
\hline & 6 & $8^{\circ} 50^{\prime} 15.144^{\prime \prime}$ & $\begin{array}{c}125^{\circ} 49^{\prime} \\
19.8948^{\prime \prime}\end{array}$ & 500.68 & \\
\hline \multirow{3}{*}{$\begin{array}{c}\text { Area } 3 \\
\text { Barangay } \\
\text { Mt.Carmel }\end{array}$} & 7 & $\begin{array}{c}8^{\circ} 49^{\prime} \\
21.7632^{\prime \prime}\end{array}$ & $\begin{array}{c}125^{\circ} 50^{\prime} \\
32.8884^{\prime \prime}\end{array}$ & 708.5 & \multirow{3}{*}{$\begin{array}{l}\text { old-growth forest, } \\
\text { secondary growth } \\
\text { forest and open- } \\
\text { areas }\end{array}$} \\
\hline & 8 & $\begin{array}{c}8^{\circ} 48^{\prime} \\
21.5748^{\prime \prime}\end{array}$ & $\begin{array}{c}125^{\circ} 49^{\prime} \\
25.9608^{\prime \prime}\end{array}$ & 550.6 & \\
\hline & 9 & $8^{\circ} 47^{\prime} 4.6172^{\prime \prime}$ & $\begin{array}{c}125^{\circ} 48^{\prime} \\
37.7064^{\prime \prime}\end{array}$ & 547.17 & \\
\hline \multirow{3}{*}{$\begin{array}{c}\text { Area } 4 \\
\text { Barangay } \\
\text { San Juan }\end{array}$} & 10 & $\begin{array}{c}8^{\circ} 51^{\prime} \\
31.3848^{\prime \prime}\end{array}$ & $\begin{array}{c}125^{\circ} 50^{\prime} \\
19.4172^{\prime \prime}\end{array}$ & 441.33 & \multirow{3}{*}{ old-growth forest } \\
\hline & 11 & $\begin{array}{c}8^{\circ} 51^{\prime} \\
34.7688^{\prime \prime}\end{array}$ & $\begin{array}{c}125^{\circ} 51^{\prime} \\
28.7208^{\prime \prime}\end{array}$ & 702.66 & \\
\hline & 12 & $\begin{array}{c}8^{\circ} 52^{\prime} \\
21.8352^{\prime \prime}\end{array}$ & $\begin{array}{c}125^{\circ} 50^{\prime} \\
53.6604^{\prime \prime}\end{array}$ & 441 & \\
\hline
\end{tabular}

Table 1. Sampling stations and the coordinates and elevations of the sampling sites in AWFR, Caraga Region, Philippines.

istic method that includes intensive searching in the different microhabitats of herpetofauna was done. Species were searched along the rocks, forest ground, trees, trunks, and branches, raking leaf litter, bodies of water, and other microhabitats (Haribon Foundation, 2001). Nine fieldworkers, searching between 0800-1200 and 1800-2200 for three consecutive days were spent for each sampling stations for a total of 816 man-hours effort.

The species accumulation curve was computed for the adequacy of the sampling effort using the software PRIMER version 6. Michaelis Menten (MM) and Ugland, Gray, Elison (UGE) were the species estimators and presented that the sampling effort was enough. The Bootstrapping procedure indicates the number of species that would be added if the sampling effort was extended. The results showed that there would possibly be five more species to be recorded if the sampling was extended (Fig. 6).

\section{Processing and identification of samples}

The collected samples were photographed, and morphometrics were taken to aid in the identification. Snout to vent length (SVL), head length (HL), head width (HW), forelimb length (FL), leg length (LL), and Tail length (TL) were measured using a vernier caliper while average body weight (BW) was measured using a portable weighing scale. Handling time was kept at a minimum to minimize 


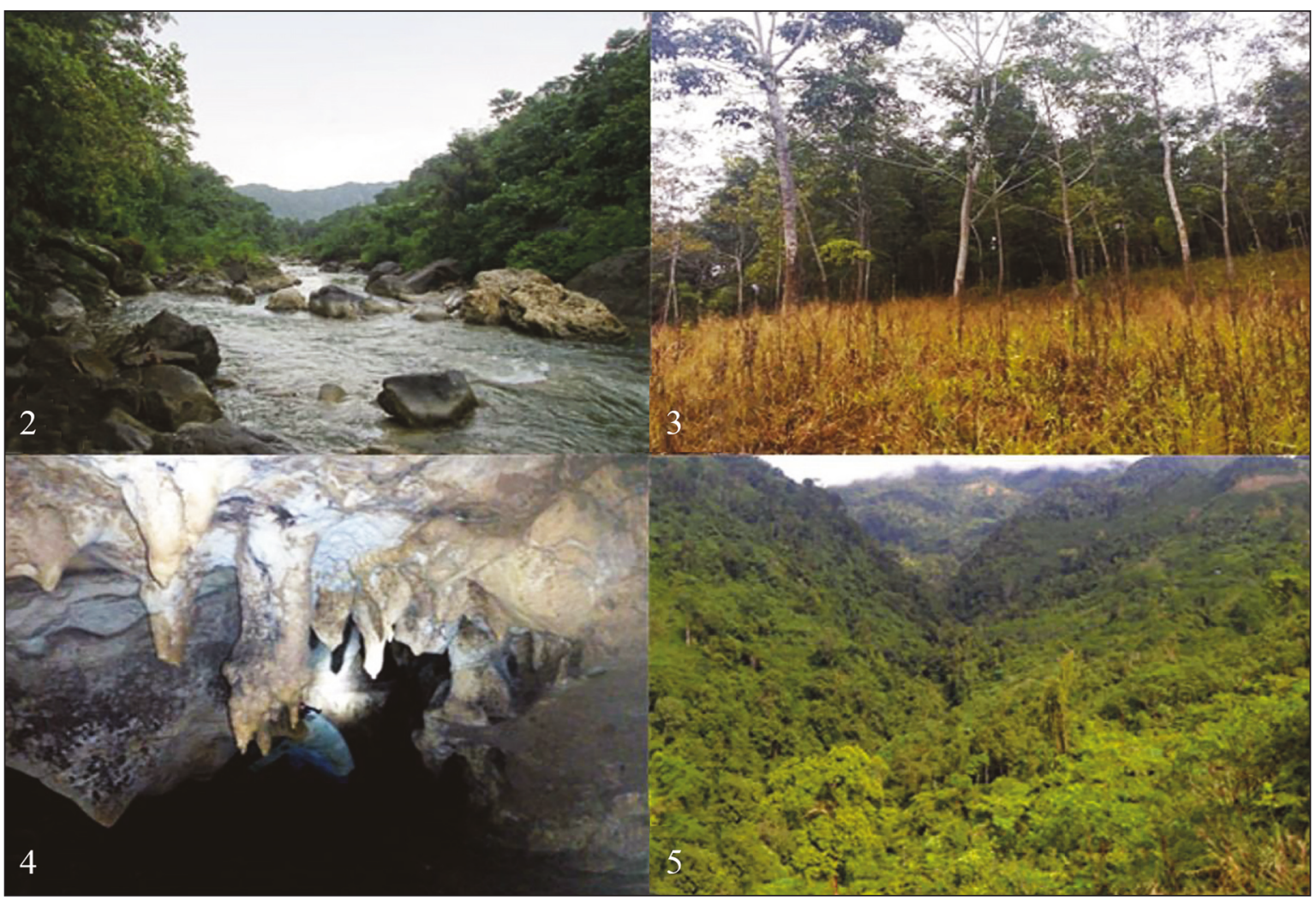

Figures 2-5. The habitats of the four sampling stations: Calaitan River (Fig. 2); plantations in Berseba (Fig. 3); one of the hundred caves in Mt. Carmel (Fig. 4); the remaining blocks of dipterocarp forest in San Juan (Fig. 5).

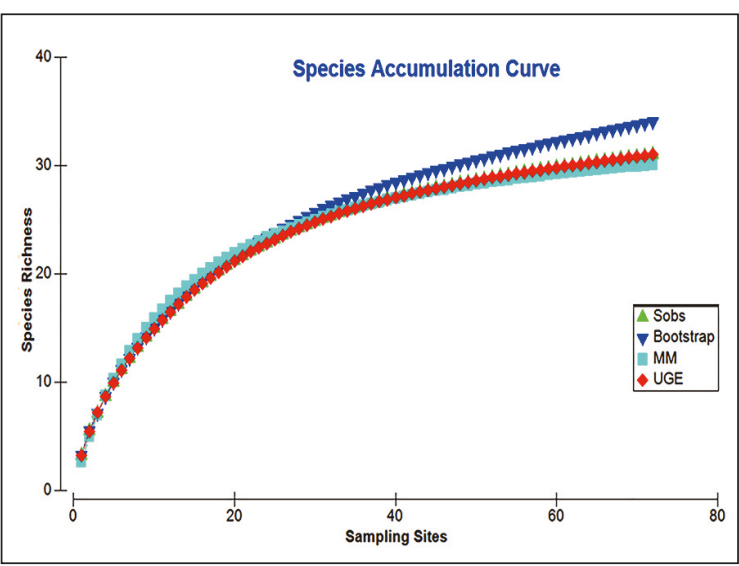

Figure 6. Species Accumulation Curve during the study period.

stress on the specimen. Collected samples were subsequently released back into the field. Before releasing the samples to the environment, species were marked with water-based paint to avoid duplication of counts when recaptured.
Samples were identified using available photographic and taxonomic key guides (Brown \& Alcala, 1980; Alcala, 1986; Nuñeza, 2012), and utilizing recently published articles on the herpetofauna in the Philippines. An expert also verified the identification of species. The conservation and distribution status were noted based on the 2018 IUCN Red List of Threatened Species and the works of Sanguila et al (2016) and Diesmos et al. (2015).

\section{Measurement of Environmental variables}

To quantitatively characterize the quality of forest which may play a role in the distribution of species, detailed measurement of environmental variables was done, based from the environmental variables used by Urbina-Cardona et al. 2006 with the addition of distance to disturbance. Physico-chemical parameters such as percent canopy cover, ground cover, herbs shrubs, and ferns and relative humidity, leaf litter depth, elevation, distance to disturbance, and air and water temperature were measured. 


\section{Data Analyses}

The statistical program $R$ version 4.0 .0 was used in determining species richness, Shannon and Simpson diversity, One-way ANOVA, and Tukey's Multiple Comparison (Pair-wise Comparison). Species Accumulation Curve, Cluster Analysis, Analysis of Similarities (ANOSIM), and Similarity Percentages (SIMPER) analysis were done using PRIMER Software version 6. Hierarchical Cluster Analysis was done to examine community structure across sites. Bray-Curtis similarity measure was used to measure site similarities based on species composition. The sites were clustered using group average. Groupings were created and represent distinct herpetofaunal composition. The lower the branch (or no branch at all) the more similar the herpetofaunal composition has been. ANOSIM was calculated to determine the Global $\mathrm{R}$ value while SIMPER Analysis was conducted to examine species contributions to similarity among sites using Bray Curtis index. Both analysis uses habitat types as factor.

Only significant environmental variables at $a<0.05$ (canopy cover and air temperature) and Philippine endemic amphibians and reptiles were used for Canonical Correspondence Analysis (CCA) using Paleontological Statistics (PAST) Software.

\section{RESULTS AND DISCUSSION}

\section{Herpetofaunal Composition}

This study was the first to report the species composition of herpetofauna in the Andanan Watershed Forest Reserve (AWFR). There were a total of 49 species of herpetofauna identified and recorded including the 22 anurans, 17 species of lizards, nine species of snakes, and one species of freshwater turtle accounting to 678 individuals (Table 2 and Figs. 7-51) in the established sampling stations of AWFR. Vulnerable species (Hydrosaurus pustulatus and Cuora amboinensis) and near threatened species (Limnonectes magnus) of the IUCN were recorded. Two introduced species of frogs were recorded ( $\mathrm{Ho}$ plobatrachus rugulosus and Rhinella marina), which were commonly seen in cultivation and open areas in the watershed. The Philippines set its own updated Red List Status through DAO 2019-09. One species captured is vulnerable (Platymantis rabori), ten species are considered Other Threatened Species, and 25 species are classified as Other Wildlife Species while the remaining 13 species were not listed in the Philippine Red List.

The Mindanao endemic and near-threatened, $L$. magnus was the most abundant anuran species $(\mathrm{n}=134)$ while the Mindanao endemic and leastconcern, E. multicarinata was the most abundant reptile $(\mathrm{n}=30)$. Five anurans (R. marina, L. magnus, $M$. stejenegeri, P. grandocula, and P. leucomystax) and four reptiles (H. frenatus, E. multicarinata, E. multifasciata, and L. s. philippinica) were consistently observed in all sampling stations of AWFR.

\section{Forest Community Types and Characteristics}

Percentage Similarity of species richness and species abundance across the surveyed sites utilizing the Bray-Curtis similarity matrix was done to show the community resemblances (Fig. 52). The result showed two main groups with a 3\% similarity. Group 1 is dominated by OFG while Group 2 is composed of Mixed habitats. The second group further branched out into two groups at $11 \%$ similarity. Group 2 sites clustered together because of similarities of species in the habitats namely; SGF, $\mathrm{CA}, \mathrm{AFH}, \mathrm{OAH}$. The cluster analysis showed that there was only less similarity in terms of species composition across all sites and species recorded among OFG sites were seen less or not found in other habitat types.

\section{Species Composition Patterns and Diversity}

Species richness was highest in the secondary growth forest with 41 species while lowest in the open-area with only eight species (Table 3 ). The old-growth forest houses 17 species, 21 in the cultivated area and 11 in the agro-forest habitats. In terms of species diversity, the secondary-growth forest still gained the highest $\left(H^{\prime}=2.65\right)$ and lowest in the openarea habitat $\left(H^{\prime}=1.81\right)$. The gamma diversity of the landscape was high, $\mathrm{H}^{\prime}=3.09$. Analysis of Variance (ANOVA) showed a highly significant difference among amphibians and reptile species in the five habitat types with an overall $\mathrm{P}<0.001$. Tukey's Multiple Comparison showed that the secondary growth forest was significantly different in terms of species composition and abundance among habitats.

Analysis of Similarity (ANOSIM) determined that the herpetofaunal community of AWFR habitats varied significantly, $\mathrm{R}=0.79$, and $P<0.001$ (Table 


\begin{tabular}{|c|c|c|c|c|c|c|c|c|}
\hline Taxa & $\begin{array}{l}\text { Distribution } \\
\text { Status }\end{array}$ & $\begin{array}{l}\text { Conser- } \\
\text { vation } \\
\text { Status }\end{array}$ & $\begin{array}{l}\text { Philippi- } \\
\text { ne Red } \\
\text { List } \\
\text { Status }\end{array}$ & $\begin{array}{l}\text { Area 1 } \\
\text { Calaitan }\end{array}$ & $\begin{array}{l}\text { Area 2 } \\
\text { Berseba }\end{array}$ & $\begin{array}{l}\text { Area } 3 \\
\text { Mt. } \\
\text { Carmel }\end{array}$ & $\begin{array}{l}\text { Area } \\
4 \text { San } \\
\text { Juan }\end{array}$ & $\begin{array}{l}\text { Total } \\
\text { Abun- } \\
\text { dance }\end{array}$ \\
\hline \multicolumn{9}{|l|}{ AMPHIBIANS } \\
\hline \multicolumn{9}{|l|}{ Bufonidae } \\
\hline Ansonia muelleri & ME & $\mathrm{LC}$ & OWS & & & & $\sqrt{ }$ & 8 \\
\hline $\begin{array}{l}\text { Pelophryne } \\
\text { brevipes }\end{array}$ & NE & $\mathrm{LC}$ & - & & & & $\sqrt{ }$ & 1 \\
\hline Rhinella marina & I & $\mathrm{LC}$ & - & $\sqrt{ }$ & $\sqrt{ }$ & $\sqrt{ }$ & $\sqrt{ }$ & 36 \\
\hline \multicolumn{9}{|l|}{ Ceratobatrachidae } \\
\hline $\begin{array}{l}\text { Platymantis } \\
\text { dorsalis }\end{array}$ & $\mathrm{PE}$ & $\mathrm{LC}$ & OWS & $\sqrt{ }$ & & & $\sqrt{ }$ & 3 \\
\hline $\begin{array}{l}\text { Platymantis } \\
\text { guentheri }\end{array}$ & $\mathrm{ME}$ & $\mathrm{LC}$ & OWS & & & $\sqrt{ }$ & & 1 \\
\hline $\begin{array}{l}\text { Platymantis } \\
\text { rabori }\end{array}$ & ME & $\mathrm{LC}$ & VU & & & & $\sqrt{ }$ & 5 \\
\hline \multicolumn{9}{|l|}{ Dicroglossidae } \\
\hline $\begin{array}{l}\text { Fejervarya } \\
\text { moodiei }\end{array}$ & $\mathrm{PE}$ & DD & OWS & $\sqrt{ }$ & & & & 6 \\
\hline $\begin{array}{l}\text { Fejervarya } \\
\text { vittigera }\end{array}$ & $\mathrm{PE}$ & $\mathrm{LC}$ & OWS & $\sqrt{ }$ & $\sqrt{ }$ & $\sqrt{ }$ & & 15 \\
\hline $\begin{array}{l}\text { Hoplobatrachus } \\
\text { rugulosus }\end{array}$ & I & LC & - & $\sqrt{ }$ & & & & 1 \\
\hline $\begin{array}{l}\text { Limnonectes } \\
\text { leytensis }\end{array}$ & $\mathrm{PE}$ & $\mathrm{LC}$ & OWS & & $\sqrt{ }$ & & $\sqrt{ }$ & 12 \\
\hline $\begin{array}{l}\text { Limnonectes } \\
\text { magnus }\end{array}$ & ME & NT & OTS & $\sqrt{ }$ & $\sqrt{ }$ & $\sqrt{ }$ & $\sqrt{ }$ & 134 \\
\hline $\begin{array}{l}\text { Occidozyga } \\
\text { laevis }\end{array}$ & $\mathrm{NE}$ & $\mathrm{LC}$ & - & & $\sqrt{ }$ & $\sqrt{ }$ & $\sqrt{ }$ & 29 \\
\hline \multicolumn{9}{|l|}{ Megophryidae } \\
\hline $\begin{array}{l}\text { Megophrys } \\
\text { stejnegeri }\end{array}$ & ME & $\mathrm{LC}$ & OTS & $\sqrt{ }$ & $\sqrt{ }$ & $\sqrt{ }$ & $\sqrt{ }$ & 45 \\
\hline \multicolumn{9}{|l|}{ Microhylidae } \\
\hline Chaperina fusca & $\mathrm{NE}$ & $\mathrm{LC}$ & - & & & $\sqrt{ }$ & & 1 \\
\hline $\begin{array}{l}\text { Kalophrynus } \\
\text { sinensis }\end{array}$ & $\mathrm{NE}$ & $\mathrm{LC}$ & - & $\sqrt{ }$ & & $\sqrt{ }$ & $\sqrt{ }$ & 10 \\
\hline $\begin{array}{l}\text { Kaloula } \\
\text { conjuncta } \\
\text { meridionalis }\end{array}$ & $\mathrm{PE}$ & $\mathrm{LC}$ & OWS & $\sqrt{ }$ & & & & 2 \\
\hline \multicolumn{9}{|l|}{ Ranidae } \\
\hline $\begin{array}{l}\text { Pulchrana } \\
\text { grandocula }\end{array}$ & ME & LC & OWS & $\sqrt{ }$ & $\sqrt{ }$ & $\sqrt{ }$ & $\sqrt{ }$ & 82 \\
\hline \multicolumn{9}{|l|}{ Rhacophoridae } \\
\hline $\begin{array}{l}\text { Kurixalus } \\
\text { appendiculatus }\end{array}$ & $\mathrm{PE}$ & LC & OWS & & & $\sqrt{ }$ & $\sqrt{ }$ & 8 \\
\hline $\begin{array}{l}\text { Philautus } \\
\text { acutirostris }\end{array}$ & $\mathrm{ME}$ & $\mathrm{LC}$ & OWS & & & $\sqrt{ }$ & $\sqrt{ }$ & 15 \\
\hline $\begin{array}{l}\text { Polypedates } \\
\text { leucomystax }\end{array}$ & $\mathrm{NE}$ & $\mathrm{LC}$ & - & $\sqrt{ }$ & $\sqrt{ }$ & $\sqrt{ }$ & $\sqrt{ }$ & 34 \\
\hline $\begin{array}{l}\text { Rhacophorus } \\
\text { bimaculatus }\end{array}$ & $\mathrm{PE}$ & $\mathrm{LC}$ & OWS & & & $\sqrt{ }$ & $\sqrt{ }$ & 11 \\
\hline $\begin{array}{l}\text { Rhacophorus } \\
\text { pardalis }\end{array}$ & $\mathrm{NE}$ & LC & - & & & & $\sqrt{ }$ & 3 \\
\hline \multicolumn{9}{|l|}{ REPTILES } \\
\hline \multicolumn{9}{|l|}{ Lizards } \\
\hline \multicolumn{9}{|l|}{ Agamidae } \\
\hline $\begin{array}{l}\text { Draco } \\
\text { cyanopterus }\end{array}$ & ME & $\mathrm{LC}$ & OWS & $\sqrt{ }$ & & & & 3 \\
\hline Draco ornatus & $\mathrm{PE}$ & $\mathrm{LC}$ & OWS & & $\sqrt{ }$ & & & 1 \\
\hline $\begin{array}{l}\text { Gonocephalus cf. } \\
\text { interruptus }\end{array}$ & $\mathrm{PE}$ & $\mathrm{DD}$ & OTS & & $\sqrt{ }$ & $\sqrt{ }$ & $\sqrt{ }$ & 5 \\
\hline $\begin{array}{l}\text { Gonocephalus cf. } \\
\text { semperi }\end{array}$ & $\mathrm{PE}$ & $\mathrm{DD}$ & OTS & & & $\sqrt{ }$ & $\sqrt{ }$ & 3 \\
\hline $\begin{array}{l}\text { Hydrosaurus } \\
\text { pustulatus }\end{array}$ & $\mathrm{PE}$ & V & OTS & & & & $\sqrt{ }$ & 4 \\
\hline
\end{tabular}




\begin{tabular}{|c|c|c|c|c|c|c|c|c|}
\hline \multicolumn{9}{|l|}{ Gekkonidae } \\
\hline $\begin{array}{l}\text { Cyrtodactylus } \\
\text { agusanensis }\end{array}$ & $\mathrm{ME}$ & $\mathrm{LC}$ & OWS & & $\sqrt{ }$ & & $\sqrt{ }$ & 5 \\
\hline $\begin{array}{l}\text { Cyrtodactylus } \\
\text { annulatus }\end{array}$ & $\mathrm{PE}$ & $\mathrm{LC}$ & OWS & & & & $\sqrt{ }$ & 2 \\
\hline Gecko gecko & $\mathrm{NE}$ & $\mathrm{LC}$ & OTS & $\sqrt{ }$ & & & & 2 \\
\hline $\begin{array}{l}\text { Hemidactylus } \\
\text { frenatus }\end{array}$ & $\mathrm{NE}$ & $\mathrm{LC}$ & - & $\sqrt{ }$ & $\sqrt{ }$ & $\sqrt{ }$ & $\sqrt{ }$ & 27 \\
\hline \multicolumn{9}{|l|}{ Scincidae } \\
\hline $\begin{array}{l}\text { Brachymeles } \\
\text { orientalis }\end{array}$ & $\mathrm{PE}$ & $\mathrm{LC}$ & OWS & & $\sqrt{ }$ & $\sqrt{ }$ & $\sqrt{ }$ & 5 \\
\hline $\begin{array}{l}\text { Eutropis } \\
\text { multicarinata }\end{array}$ & ME & $\mathrm{LC}$ & OWS & $\sqrt{ }$ & $\sqrt{ }$ & $\sqrt{ }$ & $\sqrt{ }$ & 30 \\
\hline $\begin{array}{l}\text { Eutropis } \\
\text { multifasciata }\end{array}$ & $\mathrm{NE}$ & $\mathrm{LC}$ & - & $\sqrt{ }$ & $\sqrt{ }$ & $\sqrt{ }$ & $\sqrt{ }$ & 26 \\
\hline $\begin{array}{l}\text { Lamprolepis s. } \\
\text { philippinica }\end{array}$ & $\mathrm{PE}$ & $\mathrm{LC}$ & OWS & $\sqrt{ }$ & $\sqrt{ }$ & $\sqrt{ }$ & $\sqrt{ }$ & 26 \\
\hline $\begin{array}{l}\text { Pinoyscincus } \\
\text { jagori }\end{array}$ & $\mathrm{PE}$ & $\mathrm{LC}$ & OWS & & $\sqrt{ }$ & $\sqrt{ }$ & $\sqrt{ }$ & 10 \\
\hline $\begin{array}{l}\text { Sphenomorphus } \\
\text { fasciatus }\end{array}$ & $\mathrm{PE}$ & $\mathrm{LC}$ & OWS & & $\sqrt{ }$ & $\sqrt{ }$ & $\sqrt{ }$ & 18 \\
\hline $\begin{array}{l}\text { Tropidophorus } \\
\text { misaminius }\end{array}$ & $\mathrm{ME}$ & $\mathrm{LC}$ & OWS & & $\sqrt{ }$ & $\sqrt{ }$ & $\sqrt{ }$ & 11 \\
\hline \multicolumn{9}{|l|}{ Varanidae } \\
\hline Varanus cumingi & $\mathrm{ME}$ & $\mathrm{LC}$ & OTS & $\sqrt{ }$ & & & & 1 \\
\hline \multicolumn{9}{|l|}{ Snakes } \\
\hline \multicolumn{9}{|l|}{ Colubridae } \\
\hline $\begin{array}{l}\text { Ahaetulla prasina } \\
\text { preoccularis }\end{array}$ & $\mathrm{PE}$ & $\mathrm{LC}$ & OWS & & $\sqrt{ }$ & $\sqrt{ }$ & $\sqrt{ }$ & 15 \\
\hline $\begin{array}{l}\text { Calamaria } \\
\text { lumbricoidea }\end{array}$ & $\mathrm{NE}$ & $\mathrm{LC}$ & - & & & $\sqrt{ }$ & & 1 \\
\hline $\begin{array}{l}\text { Coelognathus } \\
\text { erythrurus }\end{array}$ & $\mathrm{NE}$ & NA & OTS & $\sqrt{ }$ & & & & 1 \\
\hline $\begin{array}{l}\text { Dendrelaphis } \\
\text { philippinensis }\end{array}$ & $\mathrm{PE}$ & $\mathrm{LC}$ & OWS & & & $\sqrt{ }$ & $\sqrt{ }$ & 5 \\
\hline $\begin{array}{l}\text { Lycodon } \\
\text { capucinus }\end{array}$ & $\mathrm{NE}$ & $\mathrm{LC}$ & - & $\sqrt{ }$ & $\sqrt{ }$ & & & 2 \\
\hline \multicolumn{9}{|l|}{ Lamprophiidae } \\
\hline $\begin{array}{l}\text { Psammodynastes } \\
\text { pulverulentus }\end{array}$ & $\mathrm{NE}$ & $\mathrm{LC}$ & - & & & $\sqrt{ }$ & $\sqrt{ }$ & 5 \\
\hline $\begin{array}{l}\text { Oxyrhabdium } \\
\text { modestum }\end{array}$ & $\mathrm{ME}$ & $\mathrm{LC}$ & OWS & & $\sqrt{ }$ & $\sqrt{ }$ & $\sqrt{ }$ & 5 \\
\hline \multicolumn{9}{|l|}{ Natricidae } \\
\hline $\begin{array}{l}\text { Tropidonophis } \\
\text { dendrophiops }\end{array}$ & $\mathrm{PE}$ & $\mathrm{LC}$ & OWS & & & & $\sqrt{ }$ & 1 \\
\hline \multicolumn{9}{|l|}{ Viperidae } \\
\hline $\begin{array}{l}\text { Trimeresurus cf. } \\
\text { flavomaculatus }\end{array}$ & $\mathrm{PE}$ & $\mathrm{LC}$ & OTS & & & & $\sqrt{ }$ & 1 \\
\hline \multicolumn{9}{|l|}{ Turtle } \\
\hline \multicolumn{9}{|l|}{ Geoemydidae } \\
\hline $\begin{array}{l}\text { Cuora } \\
\text { amboinensis }\end{array}$ & $\mathrm{NE}$ & V & OTS & $\sqrt{ }$ & & & & 1 \\
\hline
\end{tabular}

Table 2. Amphibians and reptiles recorded from the four sampling stations in Andanan Watershed Forest Reserve (AWFR), Caraga Region, Philippines. Distribution and Conservation Status based on IUCN 2018: ME - Mindanao Endemic; PE Philippine Endemic; NE - Non-endemic; I - Introduced; LC - Least Concern; DD - Data Deficient; NT - Near Threatened; NA - Not Assessed; V - Vulnerable. Philippine Red List Status based on DAO2019-09: VU - Vulnerable; OTS - Other Threatened Species; OWS - Other Wildlife Species. 


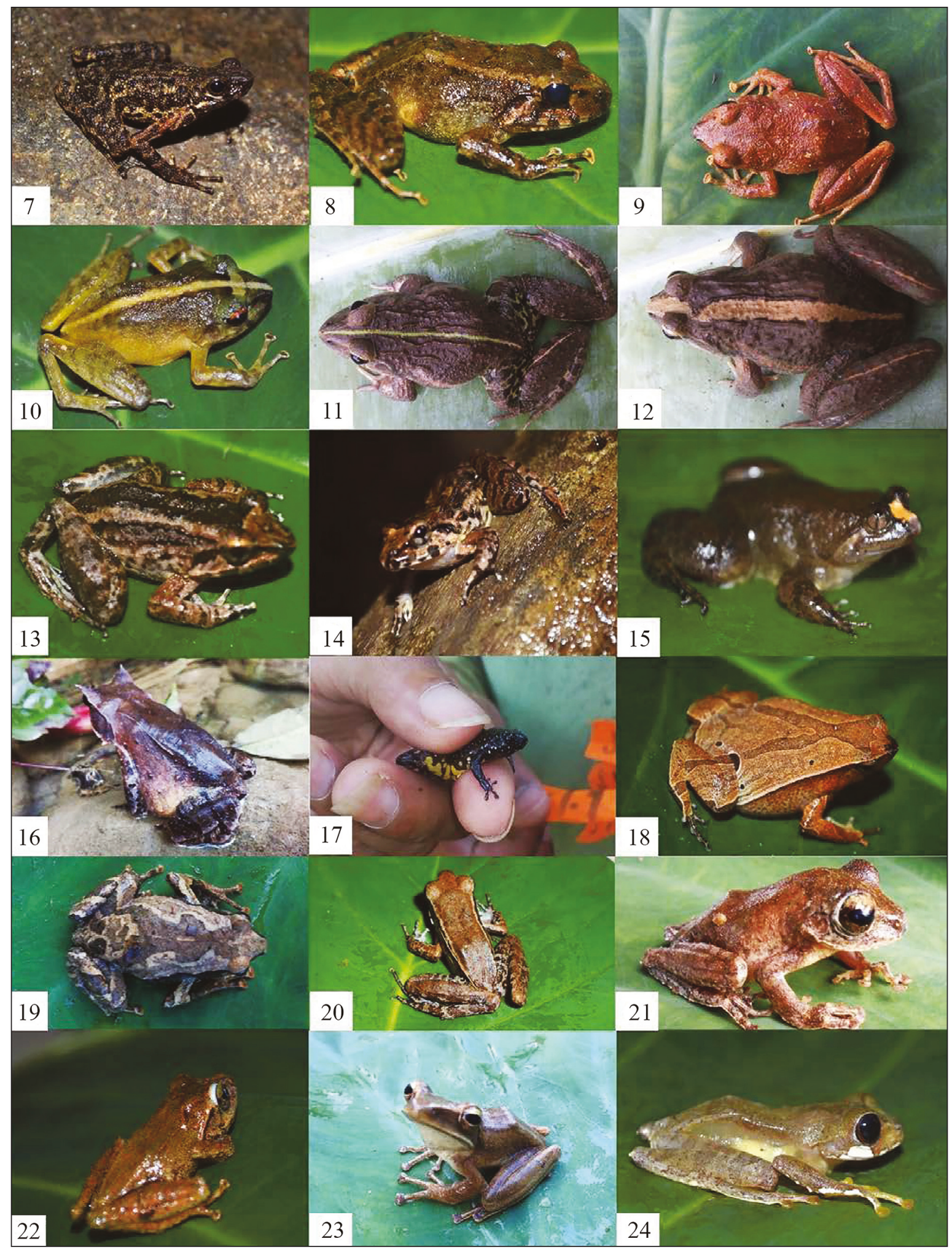

Figures 7-24. Some of the recorded amphibians in Andanan Watershed Forest Reserve: A. muelleri (Fig. 7); P. dorsalis (Fig. 8); P. guentheri (Fig. 9); P. rabori (Fig. 10); F. moodiei (Fig. 11); F. vittigera (Fig. 12); L. leytensis (Fig. 13); L. magnus (Fig. 14); O. laevis (Fig. 15); M. stejnegeri (Fig. 16); C. fusca (Fig. 17); K. sinensis (Fig. 18); K. conjuncta meridionalis (Fig. 19); P. grandocula (Fig. 20); K. appendiculatus (Fig. 21); P. acutirostris (Fig. 22); P. leucomystax (Fig. 23); R. bimaculatus (Fig. 24). 


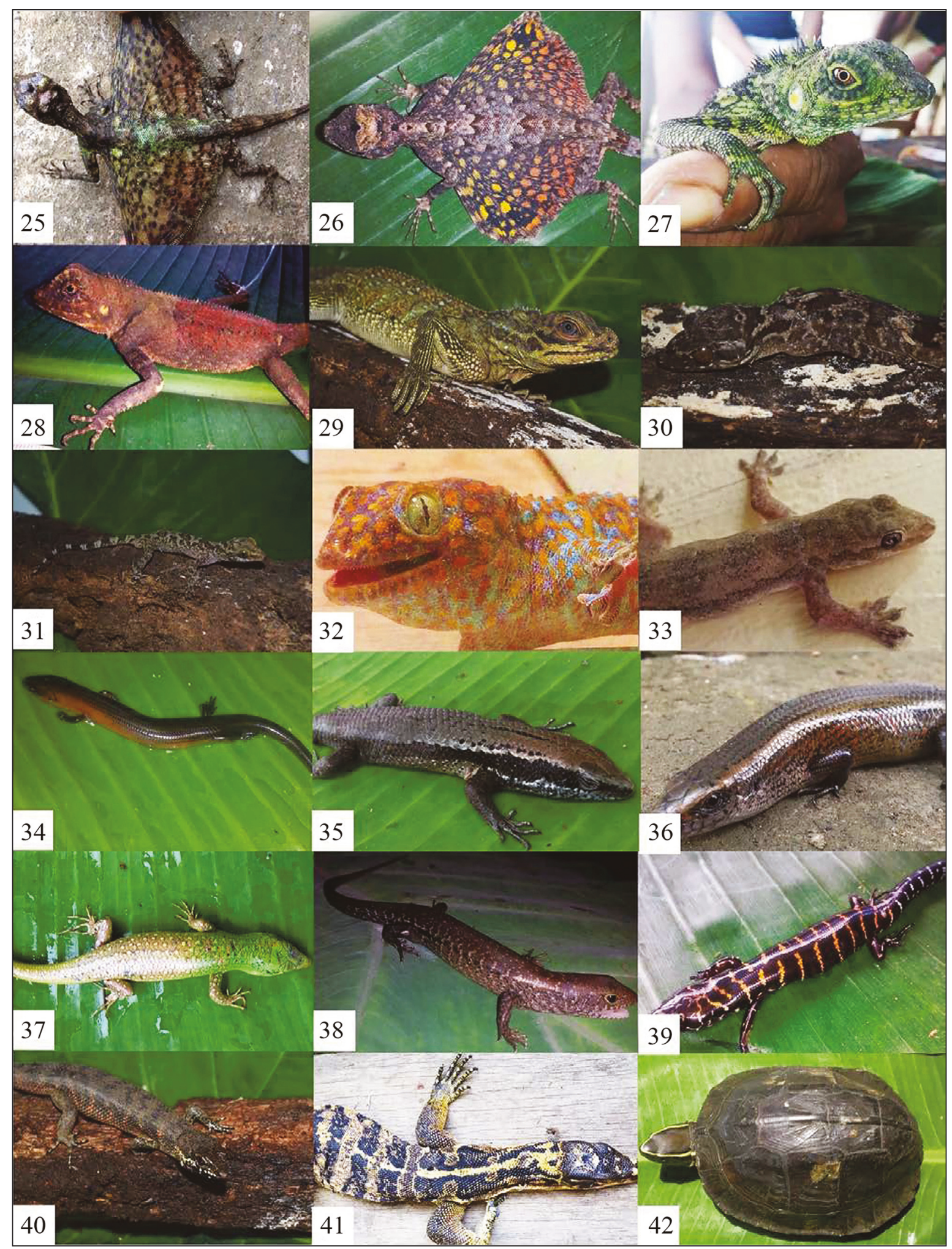

Figures 25-42. Lizards, Skinks and Turtle of AWFR: D. cyanopterus (Fig. 25); D. ornatus (Fig. 26); G. interruptus (Fig. 27); G. semperi (Fig. 28); H. pustulatus (Fig. 29); C. agusanensis (Fig. 30); C. annulatus (Fig. 31); G. gecko (Fig. 32); H. frenatus (Fig. 33); B. orientalis (Fig. 34); E. multicarinata (Fig. 35); E. multifasciata (Fig. 36); L. philippinica (Fig. 37); P. jagori (Fig. 38); S. fasciatus (Fig. 39); T. misaminius (Fig. 40);. cumingi (Fig. 41); C. amboinensis (Fig. 42). 


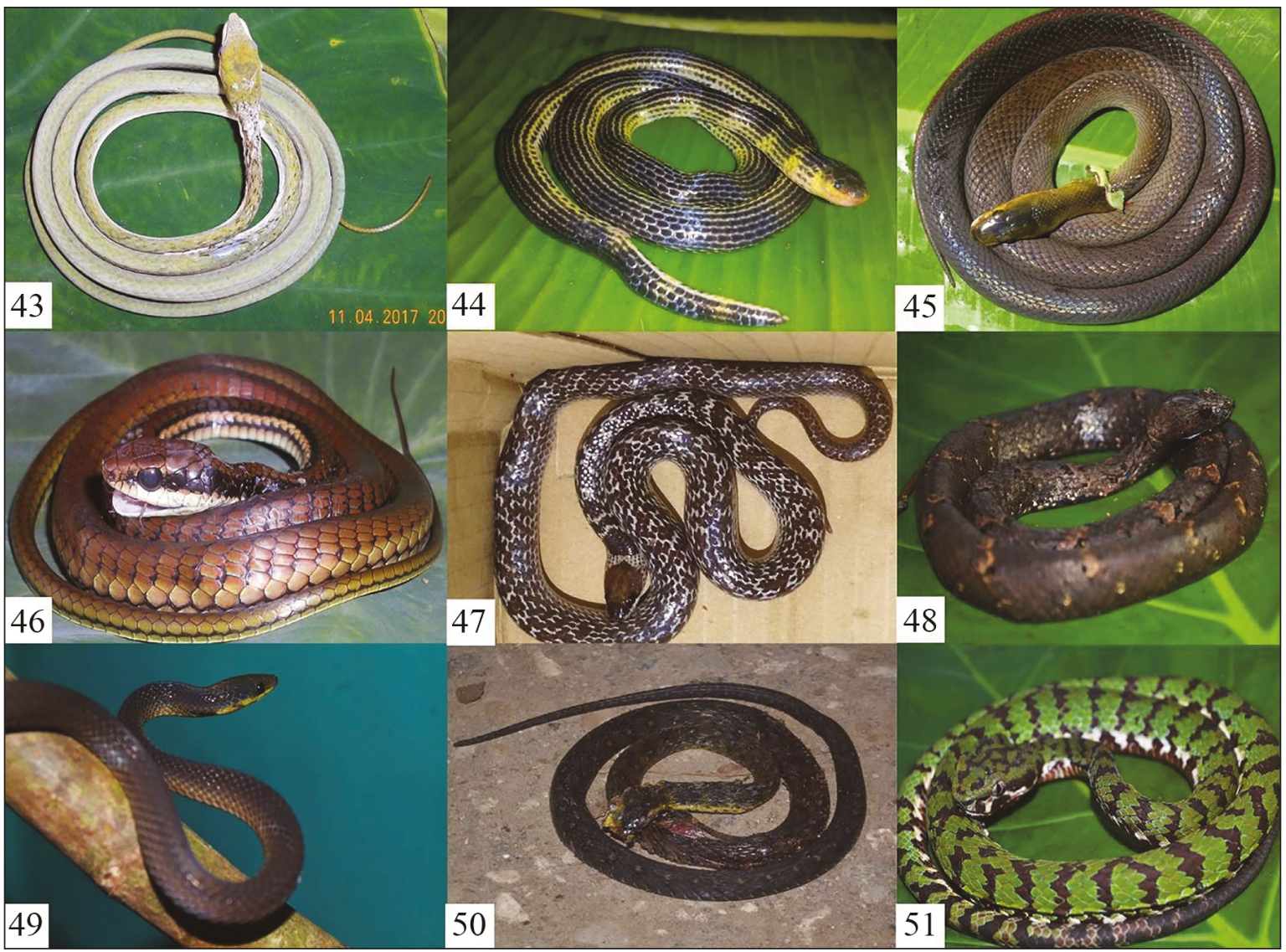

Figures 43-51. Snakes of Andanan Watershed Forest Reserve: A. prasina preoccularis (Fig. 43); C. lumbricoidea (Fig. 44); C. erythrurus (Fig. 45); D. philippinensis (Fig. 46); L. capucinus (Fig. 47); P. pulverulentus (Fig. 48); O. modestum (Fig. 49); T. dendrophiops (Fig. 50); T. flavomaculatus (Fig. 51).

\begin{tabular}{|c|c|c|c|c|c|c|}
\hline \multirow[b]{2}{*}{ Estimator } & \multicolumn{5}{|c|}{ Habitats } & \multirow[b]{2}{*}{ AWFR } \\
\hline & $\begin{array}{l}\text { Old Growth } \\
\text { Forest }\end{array}$ & $\begin{array}{c}\text { Secondary } \\
\text { Growth } \\
\text { Forest }\end{array}$ & $\begin{array}{c}\text { Cultivated } \\
\text { Area }\end{array}$ & $\begin{array}{c}\text { Agro- } \\
\text { Forested } \\
\text { Sites } \\
\end{array}$ & $\begin{array}{c}\text { Open- } \\
\text { Area }\end{array}$ & \\
\hline Species Richness & $17^{b}$ & $41^{\mathrm{a}}$ & $21^{\mathrm{b}}$ & $11^{\mathrm{b}}$ & $8^{b}$ & 49 \\
\hline $\begin{array}{l}\text { Shannon } \\
\text { Diversity (H') }\end{array}$ & 2.58 & 2.65 & 2.58 & 1.90 & 1.81 & 3.09 \\
\hline Global R value & \multicolumn{6}{|c|}{0.79} \\
\hline Overall $P$ value & \multicolumn{6}{|c|}{$<0.001 * * *$} \\
\hline
\end{tabular}

Table 3. Distribution of Herpetofauna across various habitats of AWFR, Caraga Region, Philippines. Superscript: The same letters in the line indicate insignificance at $P<0.05$; ***highly significant.

3). The dominance of several species could cause differences. The Acute-snouted tree frog dominated the old-growth forest, $P$. acutirostris, followed by the Mindanao Flying Frog, $R$. bimaculatus (SIMPER, percentage of contribution to the similarity of $38.69 \%$ and $14.45 \%$, respectively). The $L$. magnus dominated the secondary growth forest (50.56\%), while E. multicarinata dominated the cultivated area $(36.15 \%)$, A. prasina preoccularis dominated the agro-forested area $(68.80 \%)$, and $P$. leucomystax dominated the open area $(84.14 \%)$. Philautus acutirostris was an important discrim- 


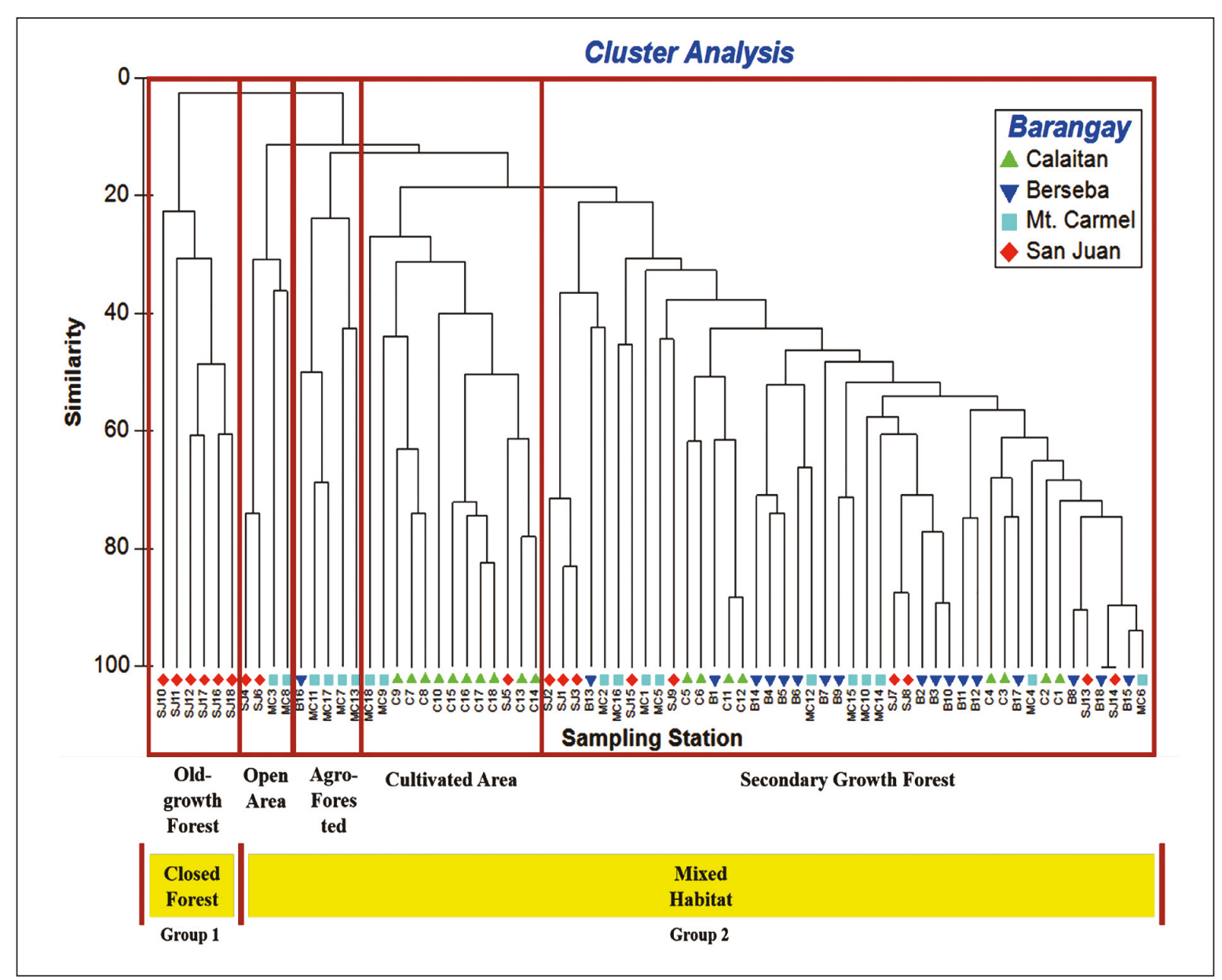

Figure 52. The percentage similarity of species richness using Bray-Curtis index of Andanan Watershed Forest reserve showing five distinct forest community types.

inatory species that dominated the old-growth forest with a percentage of contribution to the dissimilarity of $10.51 \%$ to mixed habitats (secondary growth, cultivated area, agro-forested, and open area).

\section{Herpetofaunal Species - Environment Rela- tionship}

Six of the Philippine endemic amphibian species had a strong affinity to high canopy cover (right side of the diagram). These include $P$. acutirostris, $P$. guentheri, $R$. bimaculatus, $K$. appendiculatus $P$. guentheri, and $P$. dorsalis. Other endemic species were associated with high air temperature, namely A. muelleri, L. leytensis, P. grandocula, F. vittigera, L. magnus, F. moodiei, Kaloula conjuncta meridionalis, and M. stejnegeri (Fig. 53). All Philippine endemic species of family Ceratobatrachidae (as- sociated with terrestrial microhabitats) and Rhacophoridae (associated with arboreal microhabitats) prefer closed forests that are essential for their survival. A. muelleri, although associated with high air temperature, was recorded in the open waterfall systems of the mid to high elevation in AWFR. The locally abundant and edible $L$. magnus was associated with open areas and was observed in degrading habitats.

For the reptiles, there were only eight Philippine endemic species associated with high canopy cover namely, S. fasciatus, D. philippinensis, O. modestum, B. orientalis, T. cf. flavomaculatus, T. misaminius, C. agusanensis, and P. jagori (left side of the diagram). Most of the Philippine endemic reptiles were associated with high air temperature. Flying draco (D. ornatus and D. cyanopterus) prefer open area habitats, especially coconut plantations (Fig. 54). 


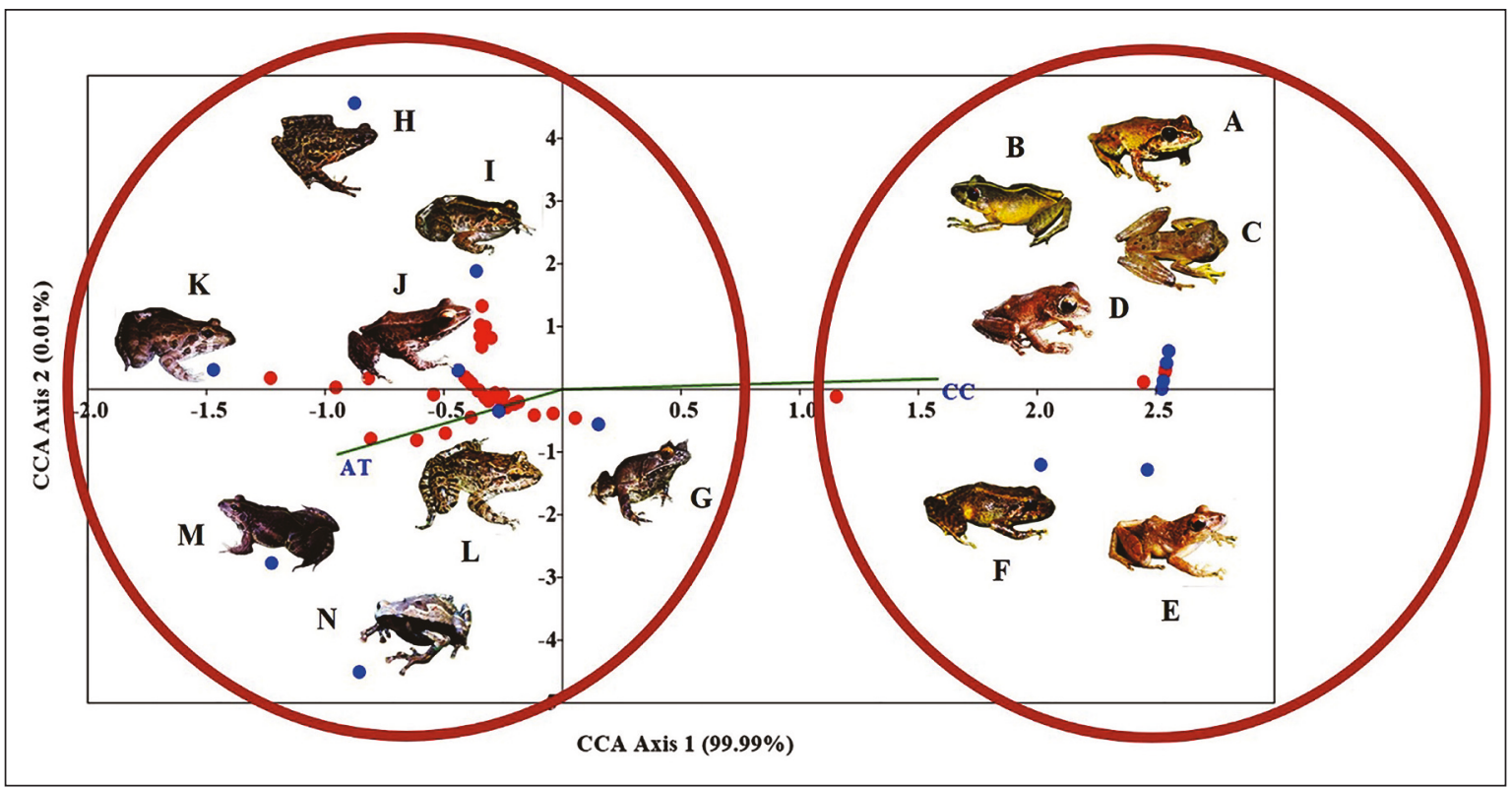

Figure 53. Ordination diagram generated by Canonical Correspondence Analysis of Philippine endemic amphibians in AWFR in relation to significant environmental traits. Blue points represent species: A. P. acutirostris, B. P. rabori, C. R. bimaculatus, D. K. appendiculatus, E. P. guentheri, F. P. dorsalis, G. M. stejnegeri, H. A. muelleri, I. L. leytensis, J. P. grandocula, K. F. vittigera, L. L. magnus, M. F. moodiei and N. Kaloula conjuncta meridionalis. Environmental traits: $\mathrm{CC}=$ Canopy cover and AT= Air Temperature. Red points represent sampling stations in AWFR.

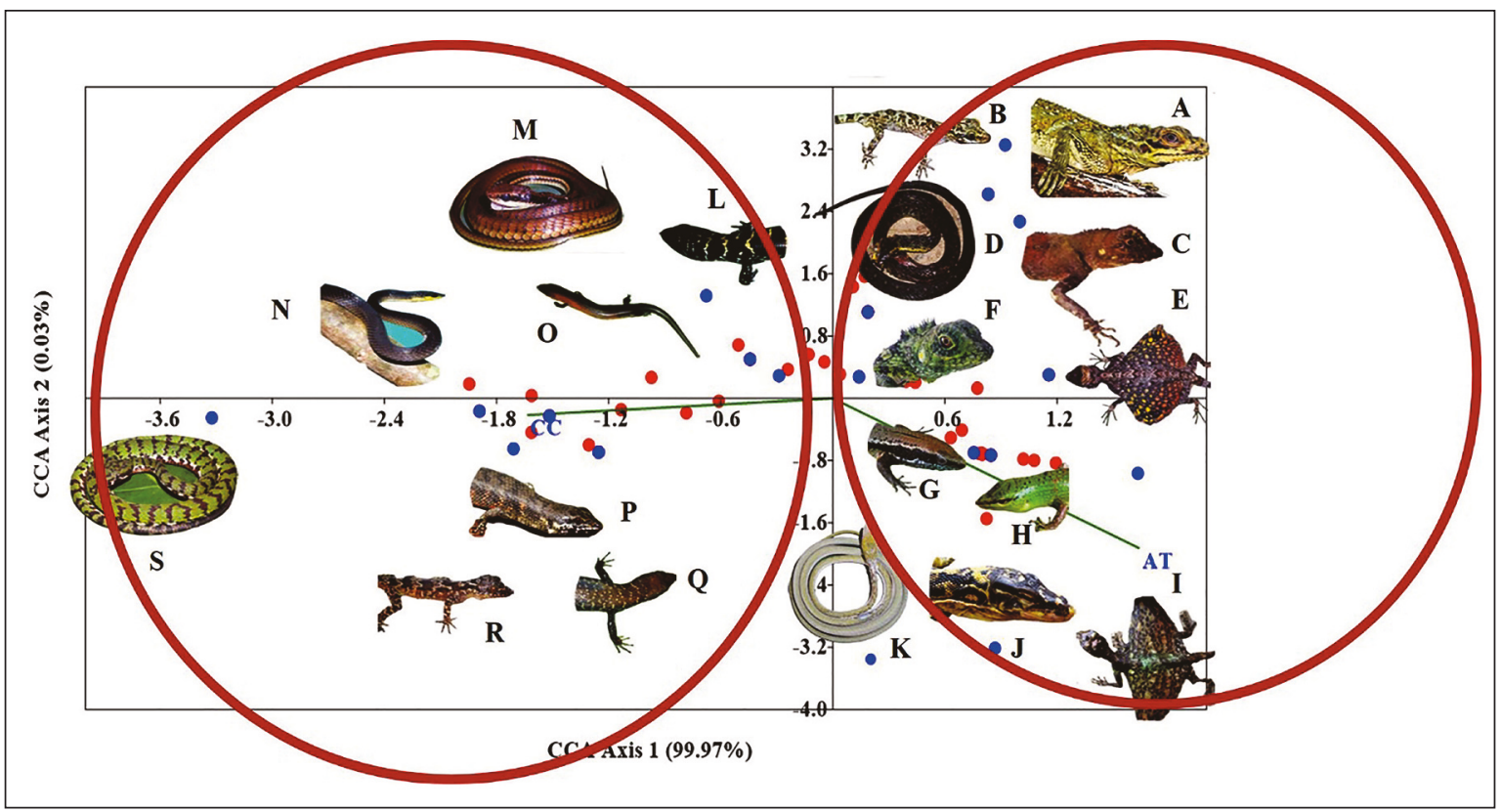

Figure 54. Ordination diagram generated by Canonical Correspondence Analysis of Philippine endemic reptiles in AWFR in relation to significant environmental traits. Blue points represent species: A. H. pustulatus, B. C. annulatus, C. G. cf. semperi, D. T. dendrophiops, E. D. ornatus, F. G. interruptus, G. E. cf. multicarinata, H. L. smaragdina philippinica, I. D. cyanopterus, J. V. cumingi, K. A. prasina preoccularis, L. S. fasciatus, M. D. philippinensis, N. O. modestum, O. B. orientalis, P. T. misaminius, Q. P. jagori, R. C. agusanensis, and S. T. cf. flavomaculatus. Environmental traits: CC= Canopy cover and $\mathrm{AT}=$ Air Temperature. Red points represent sampling stations in AWFR. 


\section{Species Accounts}

\section{Endemic Amphibians (Frogs)}

\section{Familia BUFONIDAE}

\section{Ansonia muelleri (Boulenger, 1887)}

The species is limited to montane habitats near rapids or waterfalls usually associated with high gradient streamflow (Sanguila et al., 2016). This species was observed resting above rocks near waterfalls and streams of the high elevations of Barangay San Juan, AWFR. Average morphometrics were: $\mathrm{SVL}=32.83 \mathrm{~mm}, \mathrm{HL}=10 \mathrm{~mm}, \mathrm{HW}=8.83$ $\mathrm{mm}, \mathrm{FL}=8.83 \mathrm{~mm}, \mathrm{LL}=16.17 \mathrm{~mm}$, and $\mathrm{TW}=1 \mathrm{~g}$.

\section{Familia CERATOBATRACHIDAE}

\section{Platymantis dorsalis (Duméril, 1853)}

This species inhabits forest floor stratum and is one of the most common forest amphibians in the Philippines (IUCN, 2018) with an advertisement call consisting of a pure-tone frequency sweep (Siler et al., 2007). The species recorded in the area were observed in the forest floor of Barangay Calaitan and San Juan with an average morphometrics of $\mathrm{SVL}=43 \mathrm{~mm}, \mathrm{HL}=16 \mathrm{~mm}, \mathrm{HW}=16 \mathrm{~mm}, \mathrm{FL}=10$ $\mathrm{mm}, \mathrm{LL}=23 \mathrm{~mm}$, and $\mathrm{TW}=2 \mathrm{~g}$.

\section{Platymantis guentheri (Boulenger, 1884)}

This species persists in secondary growth and fragmented habitats (Sanguila et al., 2016) and inhabits arboreal microhabitats (IUCN, 2018). The individuals recorded in AWFR were perching on vegetation just above the forest floor near a limestone cave in Mt. Carmel, AWFR. Morphometrics of the individual recorded includes $\mathrm{SVL}=42 \mathrm{~mm}$, $\mathrm{HL}=18 \mathrm{~mm}, \mathrm{HW}=17 \mathrm{~mm}, \mathrm{LL}=22 \mathrm{~mm}$, and $\mathrm{TW}=2 \mathrm{~g}$.

Platymantis rabori Brown, Alcala, Diesmos et Alcala, 1998

This uncommon species is very dependent on forest canopy for survival (Sanguila et al., 2016). It utilizes arboreal habitats in the lower montane and lowland forests (IUCN, 2018). Individuals recorded perched on trees in montane habitats of Barangay San Juan, making this species hard to detect. Aver- age morphometrics of captured individuals were: $\mathrm{SVL}=33 \mathrm{~mm}, \mathrm{HL}=14 \mathrm{~mm}, \mathrm{HW}=12 \mathrm{~mm}, \mathrm{FL}=6$ $\mathrm{mm}, \mathrm{LL}=18 \mathrm{~mm}$, and $\mathrm{TW}=1 \mathrm{~g}$.

\section{Familia DICROGLOSSIDAE}

Fejervarya moodiei (Taylor, 1920)

Fejervarya moodiei is listed as data deficient in the IUCN red list because of its unknown population distribution and biological requirements (Ohler, 2004). Individuals of this medium to large-bodied frogs were recorded from Calaitan River near agricultural sites. Average morphometrics include, $\mathrm{SVL}=65 \mathrm{~mm}, \mathrm{HL}=18 \mathrm{~mm}, \mathrm{HW}=21.5 \mathrm{~mm}, \mathrm{FL}=$ $14 \mathrm{~mm}, \mathrm{LL}=32$, and $\mathrm{TW}=10 \mathrm{~g}$.

\section{Fejervarya vittigera (Wiegmann, 1824)}

Fejervarya vittigera is known to occur in all islands of the Philippines and usually inhabits agricultural areas, ditches, and artificial ponds, and lake and flooded rice fields aggregating in large choruses (IUCN, 2018). The species is locally consumed for food because of its large hind limbs. Individuals were frequently encountered in cultivated habitats and agricultural areas of AWFR. This species was recorded in all sampling stations except in Barangay San Juan. Average morphometrics were: SVL= $64.75 \mathrm{~mm}, \mathrm{HL}=21.5 \mathrm{~mm}, \mathrm{HW}=20.88 \mathrm{~mm}, \mathrm{FL}=$ $13 \mathrm{~mm}, \mathrm{LL}=33.75 \mathrm{~mm}$, and $\mathrm{TW}=7.38 \mathrm{~g}$.

\section{Limnonectes leytensis (Boettger, 1893)}

The L. leytensis inhabits degraded and manmade environments in low elevation forests, frequently recorded on streams, rivers, and swamps (IUCN, 2018) of the Mindanao Faunal Region (Siler et al., 2009). The species were frequently encountered in rivers of secondary growth forest in Barangays Berseba and San Juan, AWFR. These species have a smaller body size compared to $L$. magnus. Average morphometric were: $\mathrm{SVL}=39.73$ $\mathrm{mm}, \mathrm{HL}=11.91 \mathrm{~mm}, \mathrm{HW}=14.27 \mathrm{~mm}, \mathrm{FL}=10 \mathrm{~mm}$, $\mathrm{LL}=20.82 \mathrm{~mm}$ and $\mathrm{TW}=1.46 \mathrm{~g}$.

\section{Limnonectes magnus (Stejneger, 1910)}

The species is locally known as 'bak-bak' and is the most abundant species captured in AWFR from disturbed lowland habitats to old-growth forests in all 
sampling stations. The species utilizes rivers, streams, wetlands, stagnant pools, agricultural, and mangrove areas. This species was listed as near threatened in the IUCN red list because of its significant declines through over-harvesting for food consumption. During the study periods, several episodes of 'pagpanulo' (the local term for hunting frogs) were observed within AWFR. Average morphometric includes: $\mathrm{SVL}=63.6$ $\mathrm{mm}, \mathrm{HL}=22.89 \mathrm{~mm}, \mathrm{HW}=22.08 \mathrm{~mm}, \mathrm{FL}=14.92$ $\mathrm{mm}, \mathrm{LL}=32.16 \mathrm{~mm}$, and $\mathrm{TW}=7.83 \mathrm{~g}$.

\section{Familia MEGOPHRYIDAE}

\section{Megophrys stejnegeri (Taylor, 1920)}

The Mindanao horned Frog is locally known as 'sungayan' and locally abundant in the area and Mindanao PAIC. It is common sitting above rocks and around the streams and river vicinity in all sampling stations of AWFR. The species is known from lowland to montane habitats (Alcala et al., 2012). It has a cryptic coloration and has pointed protrusions of skin above its eyes. The individuals were frequently encountered in slow-moving streams and near waterfalls of AWFR. Average morphometrics includes $\mathrm{SVL}=49.7 \mathrm{~mm}, \mathrm{HL}=17.75 \mathrm{~mm}, \mathrm{HW}=20.05 \mathrm{~mm}$, $\mathrm{FL}=12.8 \mathrm{~mm}, \mathrm{LL}=19.85$, and $\mathrm{TW}=7.4 \mathrm{~g}$.

\section{Familia MICROHYLIDAE}

\section{Kaloula conjuncta meridionalis Inger, 1954}

This species is restricted to lowland elevations of non-forest habitats (Alcala et al., 2012) usually in secondary growth vegetation and man-made habitats, and utilizes terrestrial and freshwater habitats (Burne \& Griffin, 2003). Individuals were recorded in leaf litters of the lowland secondary growth forests of Lake Danao, Barangay Calaitan with average morphometrics of $\mathrm{SVL}=41 \mathrm{~mm}, \mathrm{HL}=10 \mathrm{~mm}, \mathrm{HW}=$ $11 \mathrm{~mm}, \mathrm{FL}=12 \mathrm{~mm}, \mathrm{LL}=10 \mathrm{~mm}$, and $\mathrm{TW}=2 \mathrm{~g}$.

\section{Familia RANIDAE}

\section{Pulchrana grandocula (Taylor, 1920)}

Previously known as Hylarana grandocula, $P$. grandocula is a widespread and commonly encountered species of the Mindanao PAIC islands (Brown \& Guttman, 2002). It inhabits lowland to montane forest streams, rivers, and ponds (Alcala et al. 2012) and thrives from unpolluted water systems to disturbed areas. P. grandocula was the second most abundant species recorded in the area, recorded in all sampling stations of AWFR. Average morphometrics were: $\mathrm{SVL}=54.13 \mathrm{~mm}, \mathrm{HL}=19.85 \mathrm{~mm}, \mathrm{HW}=17.92$ $\mathrm{mm}, \mathrm{FL}=13.53 \mathrm{~mm}, \mathrm{LL}=27.42$, and $\mathrm{TW}=3.45 \mathrm{~g}$.

\section{Familia RHACOPHORIDAE}

\section{Kurixalus appendiculatus (Günther, 1858)}

It is a forest-dependent species mainly arboreal and uncommon to rare in nature (Alcala et al., 2012). Several individuals of this species were recorded above understory vegetation with moist ground cover in the montane forests of Mt. Carmel, AWFR. Average morphometrics were: $\mathrm{SVL}=37.5$ $\mathrm{mm}, \mathrm{HL}=13.5 \mathrm{~mm}, \mathrm{HW}=14 \mathrm{~mm}, \mathrm{FL}=8 \mathrm{~mm}, \mathrm{LL}=$ $18.5 \mathrm{~mm}$, and $\mathrm{TW}=1 \mathrm{~g}$.

\section{Philautus acutirostris (Peters, 1867)}

The $P$. acutirostris is an arboreal and terrestrial dwelling amphibian and a Mindanao endemic species commonly encountered from lowland to montane habitats (Alcala et al., 2012). Individuals of this species were captured from trees, shrubs, ferns, and terrestrial microhabitats of Mt. Carmel and San Juan forest only. The calls of these medium-sized amphibians act as cues to this undetectable species. Recorded average morphometrics include $\mathrm{SVL}=24 \mathrm{~mm}, \mathrm{HL}=9 \mathrm{~mm}, \mathrm{HW}=9 \mathrm{~mm}, \mathrm{FL}=$ $4 \mathrm{~mm}, \mathrm{LL}=13 \mathrm{~mm}$, and $\mathrm{TW}=1 \mathrm{~g}$.

\section{Rhacophorus bimaculatus (Peters, 1867)}

The uncommon tree frog, $R$. bimaculatus, inhabits arboreal microhabitats (Alcala et al., 2012). It was abundantly recorded from the montane habitats, perching above leaves of shrubs and giant ferns in the montane forests of Barangay Mt. Carmel and San Juan. Average morphometrics includes $\mathrm{SVL}=37.5 \mathrm{~mm}, \mathrm{HL}=13 \mathrm{~mm}, \mathrm{HW}=13 \mathrm{~mm}, \mathrm{FL}=$ $7.5 \mathrm{~mm}, \mathrm{LL}=21.5 \mathrm{~mm}$, and $\mathrm{TW}=1 \mathrm{~g}$.

\section{Endemic Reptiles (Lizards)}

Familia AGAMIDAE

\section{Draco cyanopterus Peters, 1867}

The species is common in secondary growth forests and cultivated areas particularly coconut 
plantations (Sanguila et al., 2016; Supsup et al., 2017). Individuals of D. cyanopterus were captured in the coconut palm and trunk in the vicinity of Lake Danao of Barangay Calaitan. Average morphometrics includes $\mathrm{SVL}=190 \mathrm{~mm}, \mathrm{HL}=15$ $\mathrm{mm}, \mathrm{HW}=12 \mathrm{~mm}, \mathrm{FL}=6 \mathrm{~mm}, \mathrm{LL}=12 \mathrm{~mm}$, and $\mathrm{TW}=5 \mathrm{~g}$.

\section{Draco ornatus (Gray, 1845)}

D. ornatus is one of the ten Philippine endemic flying lizards and a least concern (IUCN, 2018) because of its wide distribution in the country. Only one individual was collected in a coconut plantation adjacent to the secondary growth forest of Barangay Berseba. Morphometrics includes $\mathrm{SVL}=71 \mathrm{~mm}, \mathrm{HL}=12 \mathrm{~mm}, \mathrm{HW}=9 \mathrm{~mm}, \mathrm{FL}=5$ $\mathrm{mm}, \mathrm{LL}=11$, and $\mathrm{TW}=0.5 \mathrm{~g}$.

\section{Gonocephalus interruptus Boulenger, 1885}

The lizard is Mindanao endemic and treated as least concern (IUCN, 2018). Individuals were encountered resting on the tree trunks and foraging at branches of saplings in the secondary growth forest of Barangay Berseba, Mt. Carmel, and San Juan. Average morphometrics includes $\mathrm{SVL}=110 \mathrm{~mm}, \mathrm{HL}=40 \mathrm{~mm}, \mathrm{HW}=29 \mathrm{~mm}$, and $\mathrm{TW}=35 \mathrm{~g}$.

\section{Gonocephalus cf. semperi (Peters, 1887)}

Gonocephalus cf. semperi is a Philippine endemic reptile classified as least concern (IUCN, 2018). Specimens were collected during a nocturnal survey in the branches and trunk of small trees and saplings in the secondary growth forest of Barangay Mt. Carmel and San Juan. Average morphometrics includes $\mathrm{SVL}=106 \mathrm{~mm}, \mathrm{HL}=42 \mathrm{~mm}, \mathrm{HW}=31$ $\mathrm{mm}$, and $\mathrm{TW}=38 \mathrm{~g}$.

\section{Hydrosaurus pustulatus Eschsholtz, 1829}

Juveniles of $H$. pustulatus were mostly encountered and collected during nocturnal survey sleeping in rock crevices along stream beds and shrubs and saplings in Barangay San Juan. At the same time, an adult individual, approximately weighed $2000 \mathrm{~g}$, was sighted sleeping in the canopy of a tree adjacent to the same Barangay stream. Due to hunting and continuing fragmen- tation of forest in which this species is highly dependent, it is listed as vulnerable species (IUCN, 2018) for conservation purposes.

\section{Familia GEKKONIDAE}

\section{Cyrtodactylus agusanensis (Taylor, 1915)}

Individuals of this Eastern Mindanao endemic species were encountered in boulders along the banks of streams in Barangay Berseba. Other individuals were also collected in rotten logs covered with moss in the montane forest of Barangay San Juan where the stream can be found. The specimen has a SVL of $59 \mathrm{~mm}$ and a TW of $2 \mathrm{~g}$.

\section{Cyrtodactylus annulatus (Taylor, 1915)}

This gecko has been recorded from a wide variety of microhabitats, including among detritus on the forest floor, under rotting logs in forests, beneath the bark, on the surface of stumps and living trees, and in arboreal ferns (Ferner et al., 2000). Individuals were collected at night in rotten logs adjacent to streams in the montane forest of Barangay San Juan. Morphometrics include $\mathrm{SVL}=60 \mathrm{~mm}, \mathrm{TL}=$ $55 \mathrm{~mm}$ and $\mathrm{TW}=1 \mathrm{~g}$.

\section{Familia SCINCIDAE}

Brachymeles orientalis Brown et Rabor, 1967

Individuals of this skink were often encountered in agricultural areas and secondary growth forests of Barangay Berseba, Mt. Carmel, and San Juan. Species were frequently observed on the ground covered with grasses and rotten coconut husks. Because of its wide distribution, this species is classified as least concern (IUCN, 2018). The average SVL is $43 \mathrm{~mm}$, and a TW of $0.8 \mathrm{~g}$.

\section{Eutropis multicarinata (Gray, 1845)}

Eutropis multicarinata were frequently sighted basking in an open area, and mostly found sheltering in leaf litter, grasses, rotting logs, and rotting coconut husk in the riparian area of the four sampling stations. The specimen was collected in littered bamboo leaves in Barangay Calaitan. Average morphometrics includes $\mathrm{SVL}=51 \mathrm{~mm}, \mathrm{TL}=98 \mathrm{~mm}$, and $\mathrm{TW}=1.1 \mathrm{~g}$. 
Lamprolepis smaragdina philippinica (Mertens, 1928)

Lampropelis smaragdina philippinica is a Philippine endemic skink that can tolerate human disturbances. Individuals were commonly encountered in the agroforested areas of the four sampling stations and actively foraging in tree branches and trunks. Average morphometrics includes $\mathrm{SVL}=49$ $\mathrm{mm}, \mathrm{TL}=111 \mathrm{~mm}$, and $\mathrm{TW}=1.5 \mathrm{~g}$.

\section{Pinoyscincus jagori (Peters, 1864)}

The species can be found on the forest floor of older secondary forest and occupies abaca and banana plantations bordered by forest (Brown et al., 2012). Individuals of this skink were captured from Barangay Berseba and Mt. Carmel while sheltering under a rotting leaf sheath of abaca. Other individuals from San Juan were frequently encountered in the forest floor of secondary growth forest. Average morphometrics includes $\mathrm{SVL}=50 \mathrm{~mm}, \mathrm{TL}=89 \mathrm{~mm}$, and $\mathrm{TW}=2 \mathrm{~g}$.

Sphenomorphus fasciatus (Gray, 1845)

S. fasciatus individuals had been frequently found under leaf litters of the coconut tree and rotting coconut husks in the agroforested areas, secondary growth, and montane forests of Berseba, Mt. Carmel, and San Juan. Average morphometrics of individuals captures were: $\mathrm{SVL}=84 \mathrm{~mm}, \mathrm{TL}=$ $37 \mathrm{~mm}$, and $\mathrm{TW}=1 \mathrm{~g}$.

Tropidophorus misaminius Stejneger, 1908

Tropidophorus misaminius have reportedly been collected in moist soil under rotting logs and rocks, and also among rocks of stream beds, within forested areas (Brown \& Alcala, 1980). During survey periods, individuals were frequently encountered sheltering under rotting logs in the secondary growth forest of Barangay Berseba and Mt. Carmel. In Barangay San Juan, individuals were observed in boulders situated along stream beds. Average morphometrics includes $\mathrm{SVL}=75 \mathrm{~mm}$, and $\mathrm{TW}=2 \mathrm{~g}$.

Familia VARANIDAE

\section{Varanus cumingi Martin, 1839}

Varanus cumingi is a Mindanao Island endemic water monitor lizard. Though it is a widespread species, it is heavily harvested illegally for local and national consumption and the national and international pet and leather trade (Sy, 2015). The specimen collected in the study was salvaged by a local villager in a coconut plantation of Barangay Calaitan. It has a total length of $1430 \mathrm{~mm}$ and total weight of $4000 \mathrm{~g}$.

\section{Reptilia (Snakes) \\ Familia COLUBRIDAE}

\section{Ahaetulla prasina preoccularis (Taylor, 1912)}

Individuals of the species were frequently encountered at night on branches of bushes and saplings in the agroforest ecosystem and secondary growth forest of Barangay Berseba, Mt. Carmel, and San Juan. Average morphometrics includes $\mathrm{SVL}=1250 \mathrm{~mm}, \mathrm{TL}=565 \mathrm{~mm}$, and $\mathrm{TW}=30 \mathrm{~g}$.

Dendrelaphis philippinensis (Gunther, 1879)

Dendrelaphis philippinensis were often encountered at night in shrubs and saplings of secondary growth forest and agroforested areas in Barangay Mt. Carmel and San Juan. Individuals in Barangay San Juan were captured clinging to the branches of saplings along the stream bank. Average morphometrics includes: $\mathrm{SVL}=1450 \mathrm{~mm}$, and $\mathrm{TW}=146 \mathrm{~g}$.

\section{Familia LAMPROPHIIDAE}

Oxyrhabdium modestum (Duméril, 1853)

Adult individuals of $O$. modestum were encountered foraging in the forest floor, and most individuals were juveniles settling in the branches of small saplings and shrubs adjacent to streams in Barangay Berseba, Mt. Carmel, and San Juan. Average morphometrics include $\mathrm{SVL}=510 \mathrm{~mm}, \mathrm{TL}=100 \mathrm{~mm}$, and $\mathrm{TW}=98 \mathrm{~g}$.

\section{Familia NATRICINAE}

Tropidonophis dendrophiops (Gunther, 1883)

The species is common in riparian habitats with clean water and some extent of vegetation cover (Brown et al., 2012). During field study, the specimen was encountered in the vegetation of the 
montane forest in Brgy. San Juan adjacent to a small riverine system. Morphometrics include $\mathrm{SVL}=615$ $\mathrm{mm}, \mathrm{TL}=110 \mathrm{~mm}$, and $\mathrm{TW}=176 \mathrm{~g}$.

\section{Familia VIPERIDAE}

\section{Trimeresurus flavomaculatus (Gray, 1842)}

Trimeresurus flavomaculatus is a Philippine endemic pit viper. It is common at low elevations and may be encountered in a wide variety of habitats, ranging from lowland disturbed sites to mid-elevation primary tropical moist forest, and have been recorded within both agroforested and secondgrowth forest habitats (Brown et al., 2013). Only one individual was collected during the survey while clinging to the branches of saplings in the secondary growth forest of Barangay San Juan. The SVL measures $450 \mathrm{~mm}$, and TW of $155 \mathrm{~g}$.

\section{DISCUSSION}

The study recorded a high value of species richness $(S=49)$ and endemism $(67.35 \%)$. The record represents $10.36 \%$ of the entire Philippine herpetofauna (Diesmos et al., 2015; Uetz et al., 2018) and $38.89 \%$ in the northeast Mindanao records (Sanguila et al., 2016). The endemicity of the forest reserve is high, accounting for 33 species or $67.35 \%$ of the total species recorded (13 Mindanao endemic and 20 Philippine endemic). In comparison to other watersheds in the Philippines, herpetofaunal survey conducted in Pantabangan-Carranglan Watershed in Nueva Ecija recorded 59 species of herptiles, of which, 71.19\% were Philippine Endemics (GojoCruz et al., 2019). The Angat Dam Watershed, Bulacan yielded 63 herpetofaunal species (Mcleod et al., 2011). Surveys in the Bega watershed in Agusan del Sur recorded 16 reptile species and 13 amphibian species with $68.97 \%$ endemicity (Balmores \& Nuñeza, 2015; Calo \& Nuñeza, 2015). The recorded species richness in AWFR was higher compared to the nearby Bega Watershed.

Results of forest community resemblances (Fig. 52) showed that the old-growth forest was $97 \%$ dissimilar in terms of species richness and abundance to the other areas, because most of the collected species are adapted to mixed habitats. The secondary growth forest has higher species richness $(\mathrm{N}=41)$ compared to the old-growth forest $(\mathrm{N}=17)$ and other habitat types probably due to the random selection of sampling sites regarded as secondary-forest. Also, AWFR, as a whole, is dominated by secondarygrowth forest habitat. Moreover, secondary growth forest is becoming a dominant component in the tropics and even primary forest-dwelling amphibians were also recorded thriving in this regenerating forest (Dunn, 2004). Studies showed higher species richness and abundance of herpetofauna in the secondary forest than human-modified landscapes such as agriculture, pasture, and plantation (Thompson \& Donnelly, 2018). Gojo-Cruz et al. (2019) recorded a high species richness and diversity of amphibians and reptiles in complex habitats such as forest, riparian areas, and less disturbed interior areas than grasslands and agricultural sites. Also, herpetofaunal survey of Supsup et al (2020) recorded that secondary growth forest (both advanced and early secondary growth) provides the highest richness and diversity of herpetofauna followed by the old-growth forest and cultivated areas being the least. Changes in the forest structure, temperature, humidity, competition and even parasite fluctuations directly or indirectly influenced vegetation in secondary-growth (Thompson \& Donnelly, 2018). Light penetration is also abundant in secondary growth forests compared to the old-growth forest providing more vegetation and forest complexity (Herrera-Montes \& Brokaw, 2010). Thus, the complexity of regeneration among secondary growth forests makes it ideal for a diverse array of amphibians and reptiles.

The low species richness of the old-growth forest may be due to the recognized species trend, wherein higher elevations support less diversity because of reduced productivity (Rahbek, 1995; Supsup et al., 2020) and water and energy variables (Malonza, 2015) compared to the low elevation areas. Although less species richness was observed in the old-growth forest, however, this habitat harbors amphibians and reptiles that were not found in the other habitat types (such as Trimeresurus cf. flavomaculatus and Philautus acutirostris). The unique herpetofaunal species composition of the old-growth forest could be attributed to higher forest floor depth, soil moisture, organic matter, litter arthropod communities (Perry et al., 2012) and elevation (Supsup et al., 2020). Rare amphibians (with 25 percent or less chance of being encountered) were recorded in the natural forest compared to plantation forests of Dudpukuria-Dhopachari Wildlife Sanctuary and other protected areas of Bangladesh (Hasan \& Feeroz, 2014). 
Philautus acutirostris was abundantly recorded in the old-growth forest. P. acutirostris are forest dweller anurans that inhabit higher elevation areas ranging from 400-2000masl (Diesmos \& Brown, 2009). In this study, individuals of $P$. acutirostris were recorded in elevations between 600-700masl. A high number of $P$. acutirostris were also recorded by Baron et al (2019) in forest edges and forest interior of Montane Forest in Marilog District, Davao City.

Most of the recorded species in this study were endemic to the Philippines and some were even Mindanao faunal endemic. According to Nowakowski et al. (2016), species with smaller range sizes are niche specialists and are more sensitive to habitat modification than wide-ranging species. Thus, highlighting the importance of protecting Philippine endemic species.

Only Philippine endemic amphibians and reptiles were subjected to Canonical Correspondence Analysis. Variations in habitat characteristics such as canopy cover and air temperature significantly affects amphibian and reptile populations at $a<0.05$. The results conformed to Urbina-Cardona et al (2006), that amphibian and reptile assemblages had a strong relationship to canopy cover and temperature in tropical rainforest fragments of Los Tuxtlas Biosphere Reserve, Mexico. The physiologic and metabolic functions of amphibians and reptiles are regulated by ambient temperature (Angilletta et al., 2004).

Amphibian species richness had a positive relationship to grass, canopy, and emergent vegetation in the Western Aegean Region, Turkey (Baskale \& Kaya, 2009), and higher basal stands in watersheds of Arkansas (Loehle et al., 2005). Furthermore, increased vegetation cover favors arboreal anuran species that utilize this habitat as calling sites (Wells, 2007). It also protects eggs against desiccation and predation for terrestrial anurans (Hazell et al., 2001).

Eleven endemic reptile species recorded in this study were associated with high air temperature. A study conducted by Loehle et al (2005) showed that some reptile species prefer opencanopy areas and the low basal area stands because of warmer temperatures and abundant basking sites. Also, the increase of prey items such as small mammals in open canopies is one of the reasons for the abundance of reptile species in this habitat (Miller et al. 2004). Most snakes (three of five) were recorded in forest interiors which coincides with the observation in Pantabangan-Carranglan Watershed. Snakes are regarded as good indicators of environmental health (Gojo-Cruz et al., 2019). The remaining four skinks ( $S$. fasciatus, B. orientalis, T. misaminius, and $P$. jagori) and one lizard (C. agusanensis) were associated to great amounts of leaf litter associated with closed forests.

The presence of Philippine endemic and threatened herpetofaunal species in AWFR makes it an equally important watershed. Amphibians and reptiles helps maintain the stability in the ecosystem by exploiting prey that is too small to be available to more abundant species and provides high-quality food items to larger predators (Macneil et al., 2013). The impact of these organisms as both predators and prey is enhanced by the relatively high abundance and densities reached by many species in forest habitats (Campbell \& Campbell, 2001). In addition to their predator-prey dynamics roles, amphibians and reptiles have indirect impacts on forest ecosystem processes such as influencing the rates of litter decomposition through predation of soil invertebrates (Wyman, 1998) and dispersing the seeds of the plants they consume (Kimmons \& Moll, 2010). Both amphibians and reptiles are essential biological pest controllers, and some of the species are used for traditional medicines and vital source of protein in the local community. Hence, like any other forest inhabitant, these fauna should be given conservation priorities not only in AWFR but also in neighboring ecosystems.

Forest interior amphibian and reptile species are strongly affected by edge effect and habitat modifications. These species tend to avoid the microclimate fluctuations of the forest edge (Urbina-Cardona et al., 2006). The dispersal capability of herptiles is also limited compared to other vertebrates (Hillman et al., 2014) making them vulnerable to habitat changes. Furthermore, it was noted that there were several herptile species with an unknown population trend (IUCN 2018). Also, many herptiles were locally threatened based on the updated Philippine Red List status. These species tend to be more sensitive to habitat modification brought about by human-induced activities. Habitat modification may favor non-native species making them more tolerant and abundant compared to native species (Nowakowski et al., 
2016). The human population undermines the importance of herpetofauna and continues to threaten the lives of these organisms by degrading and destructing the habitat in which they are very dependent.

Timber harvesting and unmonitored hunting and overexploitation of edible amphibian and reptilian species were frequently observed in the area. The locals are also primarily dependent on the land resources of AWFR for crop production. The clearing of the forest and converting it to agricultural land is very evident in the four sampling stations. Oldgrowth forests were converted into cultivated areas and agricultural farms for livelihood were observed even at high elevations. These anthropogenic activities threaten the abundance of the amphibians and reptiles in the watershed.

Habitat loss due to the conversion of forest into farmland is among the most common threats observed in some of the protected areas in Mindanao (Nuñeza \& Galorio, 2015; Nuñeza et al., 2016), since the indigenous population of the corridor forests are being integrated into the mainstream of lowland culture, and are now adopting destructive lowland farming techniques in the uplands (CEPF, 2011). Also, the agricultural chemicals and pesticides used in crop production could result in secondary problems for herpetofaunal communities with the mass killing of insect communities, which was an integral component of their food niche $(\mathrm{Su}-$ rasinghe \& Jayaratne, 2006).

Since different plant species provide different ambient temperature, different prey availability, different microhabitat, and different camouflage opportunity (Valentine et al., 2007), planting variety of plant species (mainly native flora) could help in preserving the remaining forest blocks of AWFR, thus, providing an ambient environment to herpetofauna. Canopy removal should be managed appropriately within the watershed as reductions in canopy cover could cause changes in temperature, humidity and wind exposure and could also result in the temporary reduction or elimination of leaf litter inputs (Todd \& Andrews, 2008; Greenberg et al., 2011), which may negatively affect amphibian and reptilian species which are dependent with litter depth (Moorman et al., 2011). The habitat association analysis results will also help in the local conservation of the endemic species as different areas provide different microhabitats for the species.

\section{CONCLUSIONS}

High species richness, endemism, and diversity were recorded in Andanan Watershed Forest Reserve. Endemic amphibians and reptiles were significantly associated with canopy cover and air temperature. The herpetofaunal composition of the study area is only partially known and the herpetofaunal species diversity is threatened by habitat modification caused by anthropogenic disturbance. Continued surveys on the unknown portions of AWFR may account for new records of herpetofauna's diversity and endemicity in the area. It is important to protect all habitats to ensure the survival of herpetofauna present in the area. Thus, it is highly recommended to have a regular monitoring of amphibians and reptiles and their ecology and taxonomy, including various flora and fauna covering the vast portion of the unexplored area in the watershed.

\section{ACKNOWLEDGMENTS}

The authors are grateful to the Protected Area Management Board (PAMB) of Community Environment and Natural Resources Office (CENRO) and DENR Caraga for the resolution and gratuitous permit granting us to conduct the study in AWFR. The City ENRO, local government of Bayugan City, Barangay Captains, Barangay Kagawads, BPATS, and the local guides during field sampling are highly acknowledged for the logistical and safety measures they provided during the sampling periods. Dr. Rafe M. Brown is also acknowledged for verifying the identification of the specimens.

Declaration of competing interest. The authors declare that they have no known competing financial interests or personal relationships that could have appeared to influence the work reported in this paper.

\section{REFERENCES}

Alcala A.C., 1986. Guide to Philippine Flora and Fauna. Amphibians and Reptiles. Natural Resources Management Center, Ministry of Natural Resources and University of the Philippines.

Alcala A.C., Bucol A.A., Diesmos A.C., \& Brown R.M., 2012. Vulnerability of Philippine Amphibians to Cli- 
mate Change. Philippine Journal of Science, 141: 77-87.

Angilletta M.J., Steury T.D., \& Sears M.W., 2004. Temperature, growth, and body size in ectotherms: fitting pieces of a life-history puzzle. Integrative and Comparative Biology, 44: 498-509.

Balmores M.N., \& Nuñeza O.M., 2015. The Reptiles of Bega Watershed of the Province of Agusan del Sur in the Philippines. World Journal of Environmental Biosciences, 4: 50-61.

Baron-Delima E.M., Mohagan A.B., Leano E.P., \& Amoroso V.B., 2019. Philautus (Bush Frogs) Species from Montane Forest of Marilog District, Davao City, Southern Mindanao, Philippines. Environment and Natural Resources Journal, 17: 62-70.

Baskale E. \& Kaya U., 2009. Richness and Distribution of Amphibian Species in Relation to Ecological Variables in Western Aegean Region of Turkey. Ekoloji, 18: $25-31$

Beukema W., 2011. Herpetofauna of Disturbed Forest Fragments on the Lower Mt. Kitanglad Range, Mindanao Island, Philippines. Salamandra, 47: 90-98.

Brown W.C. \& Alcala A.C., 1980. Philippine Lizards of the Family Scincidae. 1st ed. Silliman University.

Brown R.M. \& Guttman S.I., 2002. Phylogenetic systematics of the Rana signata complex of Philippine and Bornean stream frogs: Reconsideration of Huxley's modification of Wallace's Line at the OrientalAustralian faunal zone interface. Biological Journal Linnean Society, 76: 393-461.

Brown R.M., Oliveros C.H., Siler C.D., Fernandez J.B., Welton L.J., Buenavente P.A.C., Diesmos M.L.L. \& Diesmos A.C., 2012. Amphibians and Reptiles of Luzon Island (Philippines), VII: Herpetofauna of Ilocos Norte Province, Northern Cordillera Mountain Range. Check List, 8: 469-490.

Brown R.M., Siler C.D., Oliveros C.H., Welton L.J., Rock A., Swab J., Van Weerd M., Van Beijnen J., Jose E., Rodriguez D. \& Diesmos A.C., 2013. The Amphibians and Reptiles of Luzon Island, Philippines, VIII: The Herpetofauna of Cagayan and Isabela Provinces, Northern Sierra Madre Mountain Range. Zoo Keys, 266: 1-120.

Burne M.R. \& Griffin C.R., 2003. Habitat Associations of Pool-breeding Amphibians in Eastern Massachusetts, USA. Wetlands Ecology and Management, 13: 247-259.

Calo T.J.V. \& Nuñeza O.M., 2015. Species richness and endemism of anurans in Bega Watershed, Prosperidad, Agusan del Sur, Philippines. Journal of Biodiversity and Environmental Sciences 7:1-14.

Campbell K.R. \& Campbell T.R., 2001. The Accumulation and Effects of Environmental Contaminants on Snakes: A Review. Environmental Monitoring and Assessment, 70: 253-301.
Critical Ecosystem Partnership Fund (CEPF), 2011. The Philippines Hotspot. Annual Report: 1-32.

Delima E.M.M., Diesmos A.C. \& Ibañez, J.C., 2007. The Herpetofaunal Importance of Mt. Hamiguitan Range, Mindanao Island, Philippines. Banwa 4: 27-40.

Diesmos A.C., Brown R.M., Alcala A.C., Sison R.V., Afuang L.E. \& Gee G.V.A., 2002. Philippine Amphibians and Reptiles: An overview of Species Diversity, Biogeography, and Conservation.

Diesmos A.C. \& Brown R.M., 2009. Diversity, Biogeography and Conservation of Philippine Amphibians. Proceedings of the Conference "Biology of the Amphibians in the Sunda Region, South-east Asia, pp. 26-49.

Diesmos A.C., Watters J.L., Huron N.A., Davis D.R., Alcala A.C., Crombie R.I., Afuang L.E., Gee-Das G., Sison R.V., Sanguila M.B., Penrod M.L., Labonte M.J., Davey C.S., Leone E.A., Diesmos M.L., Sy E.Y., Welton L.J., Brown R.M. \& Siler C.D., 2015. Amphibians of the Philippines Part I: Checklist of the Species. Proceedings of the California Academy and Science, 62: 1-84.

Dunn R.R., 2004. Recovery of faunal communities during tropical forest regeneration. Conservation Biology, 18: 302-309.

Ferner J.W., Brown R.M., Sison R.V. \& Kennedy R.S., 2000. The Amphibians and Reptiles of Panay Island, Republic of the Philippines. Asiatic Herpetological Research 9: 34-70.

FMB-DENR., 2013. Philippine Forests Facts and Figures. Forest Management Bureau - Department of Environment and Natural Resources, Visayas Avenue, Quezon City, Philippines.

Gojo-Cruz P.H.P., Afuang L.E., Gonzales J.C.T. \& Gruezo W.S.M., 2019. Distribution and diversity patterns of herpetofauna in the Pantabangan-Carranglan Watershed, Nueva Ecija, Caraballo Mountain Range, Philippines. Biodiversity Data Journal, 7: e31638.

Greenberg C.H., Collins B., Thompson F.R. III \& Mcnab W.H., 2011. What are Early Successional Habitats, Why are they Important, and How can they be Sustained? Springer, 1-10.

Hasan M.K. \& Feeroz M.M., 2014. Species Diversity and Habitat Preferences of Amphibian Fauna in six protected areas of Bangladesh. Bangladesh Journal of Zoology, 42: 105-116.

Hazell D., Cunningnham R., Lindenmayer D., Mackey B. \& Osborne W., 2001. Use of farm dams as frog habitat in an Australian agricultural landscape: factors affecting species richness and distribution. Biological Conservation, 102: 155-169.

Herrera-Montes A. \& Brokaw N., 2010. Conservation value of tropical secondary forest: A herpetofaunal perspective. Biological Conservation, 143: 14141422.

Hillman S.S., Drews R.C., Hedrick M.S. \& Hancock 
T.V., 2014. Physiological vagility and its relationship to dispersal and neutral genetic heterogeneity in vertebrates. The Journal of Experimental Biology, 217: 3356-3364.

IUCN, 2018. International Union for the Conservation of Nature, Red List of Threatened Species. Available at: https://www.iucn.redlist.org. [Date accessed: 31 December 2018].

Loehle C., Wigley T.B., Shipman P.A., Fox S.F., Rutzmoser S., Thill R.E. \& Melchiors A., 2005. Herpetofaunal species richness response to forest landscape structure in Arkansas. Forest Ecology and Management, 209: 293-308.

Kimmons J.B., \& Moll D., 2010. Seed Dispersal by RedEared Sliders (Trachemys scripta elegans) and Common Snapping Turtles (Chelydra serpentina). Chelonian Conservation and Biology, 9: 289-294.

Macneil J., Macgowan B.J., Currylow A. \& Williams R.A., 2013. Forest Management for Reptiles and Amphibians: A Technical Guide for the Midwest. Purdue Extension, 1-23.

Malonza P.K., 2015. Patterns of reptile and amphibian species richness along elevational gradients in $\mathrm{Mt}$. Kenya. Dongwuxue Yanjiu, 36: 342-347.

Manolis S.C., Webb G.J. \& Britton A.R., 2002. Crocodilians and other Reptiles: Bioindicators of Pollution. Wildlife Management International, PO Box 530 Sanderson, NT 0813, Australia, pp. 65-69.

Mcleod D.S., Siler C.D., Diesmos A., Diesmos M.L., Garcia V.S., Arkonceo A.O., Balaquit K.L., Uy C.C., Villaseran M.M., Yarra E.C. \& Brown R.M., 2011. Amphibians and Reptiles of Luzon Island, V: The Herpetofauna of Angat Dam Watershed, Bulacan Province, Luzon Island, Philippines. Asian Herpetological Research, 2: 177-198.

Miller D.A., Thill R.E., Melchiors M.A., Wigley T.B. \& Tappe P.A., 2004. Small mammal communities of streamside management zones in intensively managed pine forests of Arkansas. Forest Ecology Management, 203: 381-393.

Mindanao Protected Area Network., 2018. The Mindanao Protected Area Management Board Network. Available at: http://mindanaopamb.buksu.edu.ph/pamb/spe cific/44. [Date accessed: 30 September 2020].

Moorman C.E., Russell K.R. \& Greenberg C.H., 2011. Reptile and Amphibian Response to Hardwood Forest Management and Early Successional Habitats. Springer: 191-208.

Nowakowski A.J., Thompson M.E., Donnelly M.A. \& Todd B.D., 2016. Amphibian sensitivity to habitat modification is associated with population trends and species traits. Global Ecology and Biogeography, 26: 700-712.

Nuñeza, O.M., 2012. Guide to Amphibians and Reptiles of Mindanao, Philippines. Office of Publication and
Information OVCRE, Mindanao State University-Iligan Institute of Technology, Iligan City, Philippines, pp. 1-72.

Nuñeza O.M. \& Galorio A.H.N., 2015. Cave Herpetofauna of Siargao Island Protected Landscape and Seascape. World Journal of Environmental Biosciences, 4: $26-35$.

Nuñeza O.M., Non M.L.P., Oconer E.P. \& Aljibe M.C., 2016. Reptile Diversity in Mt. Matutum Protected Landscape, South Cotabato, Philippines. Journal of Biodiversity and Environmental Studies, 8: 9-21.

Ohler A., 2004. Fejervarya moodiei. The IUCN Red List of Threatened Species.

http://dx.doi.org/10.2305/IUCN.UK.2004.RLTS.T582 76A11748038.en.

Perry D.A., Griffiths R.P., Moldenke A.R. \& Madson S.L., 2012. Abiotic and Biotic Soil Characteristics in Old Growth Forests and Thinned or Unthinned Mature Stands in Three Regions of Oregon. Diversity, 4: 334-362.

Rahbek C., 1995. The elevational gradient of species richness: a uniform pattern? Ecography, 18: 200-205.

Relox R.E., Leano E.P. \& Camino F.B., 2011. Herpetofaunal Endemism and Diversity in Tropical Forests of Mt. Hamiguitan in the Philippines. Herpetological Conservation and Biology, 6: 107-113.

Riyanto A., 2011. Herpetofaunal community structure and habitat associations in Gunung Ciremai National Park, West Java, Indonesia. Biodiversitas, 12: $38-44$.

Sanguila M.B., Cobb K.A., Siler C.D., Diesmos A.C., Alcala A.C. \& Brown R.M., 2016. The Amphibians and Reptiles of Mindanao Island, Southern Philippines, II: The Herpetofauna of Northeast Mindanao and Adjacent Islands. ZooKeys, 624: 1-132.

Siler C.D., Linkem C.W., Diesmos A.C. \& Alcala A.C., 2007. A New Species of Platymantis (Amphibia: Anura: Ranidae) from Panay Island, Philippines. Herpetologica, 63: 351-364.

Siler C.D., McVay J.D., Diesmos A.C. \& Brown R.M., 2009. A New Species of Fanged Frog, Genus Limnonectes (Amphibia: Anura: Dicroglossidae) from Southeast Mindanao Island, Philippines. Herpetologica, 65: 105-114.

Solania C.L. \& Gamalinda E.F., 2018. Species Composition and Habitat Association of Anurans within Water Systems of Andanan Watershed, Agusan del Sur, Caraga Region, Philippines. Environmental and Experimental Biology, 16: 159-168.

Supsup C.E., Puna N.M., Asis A.A., Redoblado B.R., Panaguinit M.F.G., Guinto F.M., Rico E.B., Diesmos A.C., Brown R.M. \& Mallari N.A.D., 2016. Amphibians and Reptiles of Cebu, Philippines: The Poorly Understood Herpetofauna of an Island with Very Little Remaining Natural Habitat. Asian Herpetological Research, 7: 151-179. 
Supsup C.E., Asis A.A., Carestia Jr. U., Diesmos A.C., Mallari N.A.D. \& Brown R.M., 2020. Variation in species richness, composition and herpetological community structure across a tropical habitat gradient of Palawan Island, Philippines. Herpetozoa, 33: 95-111.

Sumanasekara V.D.W., Dissanayake D.M.M.R. \& Senevirante H.T.J., 2015. Review on use of amphibian taxa as a bio-indicator for watershed health and stresses. NBRO Symposium, Innovations for Resilient Environment.

Surasinghe T.D. \& Jayaratne R.L., 2006. Diversity, Threats and Conservation of Herpetofauna in and Around Eastern Sinharaja. Sabaragamuwa University Journal, 6: 3-12.

Sy E.Y., 2015. Checklist of exotic species in the Philippine pet trade, II. Reptiles. Journal of Nature Studies, 14: 66-93.

Thompson M.E. \& Donnelly M.A. 2018. Effects of Secondary Forest Succession on Amphibians and Reptiles: A Review and Meta-Analysis. Copeia, 106: $10-19$.
Todd B.D. \& Andrews K.M., 2008. Response of a Reptile Guild to Forest Harvesting. Conservation Biology, 22: 753-761.

Uetz P., Freed P. \& Hošek J., 2018. The Reptile Database. Available at: http://www.reptile-database.org. [Date accessed: 30 September 2020].

Valencia-Aguilar A., Cortes-Gomez A. \& Ruiz-Agudelo C.A., 2013. Ecosystem services provided by amphibians and reptiles in Neotropical ecosystem. International Journal of Biodiversity Science, Ecosystem Services \& Management, 9: 257-272.

Valentine L.E., Roberts B. \& Schwarzkopf F., 2007. Mechanisms Driving Avoidance of Non-native Plants by Lizards. Journal of Applied Ecology, 44: 228-237.

Wells K.D., 2007. The Ecology and behavior of amphibians. The University of Chicago Press, Chicago, $1148 \mathrm{pp}$.

Wyman R.L., 1998. Experimental Assessment of Salamanders as Predators of Detrital Food Webs: Effects on Invertebrates, Decomposition and the Carbon Cycle. Biodiversity and Conservation, 7: 641-650. 